TITLE:

\title{
Optimization of shared autonomous electric vehicles operations with charge scheduling and vehicle-to-grid
}

\section{$\operatorname{AUTHOR}(\mathrm{S}):$}

lacobucci, Riccardo; McLellan, Benjamin; Tezuka, Tetsuo

\section{CITATION:}

lacobucci, Riccardo ... [et al]. Optimization of shared auto nomous electric vehicles operations with charge scheduling and vehicle-to-grid. Transportation Research Part C: Emerging Technologies 2019, 100: 34-52

\section{ISSUE DATE:}

2019-03

URL:

http://hdl.handle.net/2433/259766

\section{RIGHT:}

(c) 2019. This manuscript version is made available under the CC-BY-NC-ND 4.0 license

http://creativecommons.org/licenses/by-nc-nd/4.0/:; The full-text file will be made open to the public on 1 March 2021 in accordance with publisher's 'Terms and Conditions for Self-Archiving'.; This is not the published version. Please cite only the published version.; この論文は出版社版でありません。引用の際には出版社版をご確認ご利用ください。 


\title{
Optimization of Shared Autonomous Electric Vehicles Operations with Charge Scheduling and Vehicle-to-Grid
}

\author{
Riccardo Iacobucci*, Benjamin McLellan, Tetsuo Tezuka \\ Graduate School of Energy Science, Kyoto University, Yoshida Honmachi, Sakyo-ku, \\ Kyoto 606-8501, Japan
}

\begin{abstract}
Shared autonomous electric vehicles, also known as autonomous mobility on demand systems, are expected to become commercially available by the next decade. In this work we propose a methodology for the optimization of their charging with vehicle-to-grid in parallel with optimized routing and relocation. The methodology presented is based on previous work expanded to include charge optimization. The proposed model optimizes transport service and charging at two different time scales by running two modelpredictive control optimization algorithms in parallel. Charging is optimized over longer time scales to minimize both approximate waiting times and electricity costs. Routing and relocation are optimized at shorter time scales to minimize waiting times, with the results of the long-time-scale optimization as charging constraints. This approach allows efficient optimization of both aspects of system operation. The problem is solved as a mixed-integer linear program. A case study using transport and electricity price data for Tokyo is used to test the model. Results show that the system can substantially
\end{abstract}

\footnotetext{
${ }^{*}$ Corresponding author. E-mail: iacobucci.riccardo.38e@kyoto-u.jp
} 
reduce charging costs without significantly affecting waiting times, with cost reduction dependent on electricity price variability. Vehicle-to-grid is shown to be unsuitable for current electricity and battery prices, however offering substantial savings with price profiles with higher variability.

Keywords: shared transportation, autonomous vehicles, electric vehicles, charge scheduling, model predictive control

\section{Introduction}

New models of transportation are emerging. One-way car sharing services, in which cars can be taken from wherever they are currently parked and left at any other place within a designated area, are already popular in large cities in Europe (Boyacı et al., 2015). Autonomous driving technology could speed up the adoption of this transport mode, making it more convenient as vehicles can move autonomously to pick up passengers (Fagnant and Kockelman, 2014). Autonomous driving can also lead to much more efficient energy use, further driving adoption of this technology (Vahidi and Sciarretta, 2018). The advent of shared autonomous transportation has significant implications for the energy system, since it could allow a faster electrification of the transport sector and enable a more efficient control of the charging of vehicles and large scale demand response.

Shared autonomous electric vehicles (SAEVs) represent an important opportunity for adding significant storage to the grid in a time when intermittent renewable energy penetration is rising. If correctly managed, SAEVs can offer grid-scale load shifting, peak shaving, and ancillary services. This would enable a higher renewable energy penetration and a more resilient 
electric grid. The alternative of uncoordinated charging may compromise grid stability and increase grid congestion and peak load.

It is therefore essential to optimize and coordinate SAEVs charging. However, most of the literature on the charging of electric vehicles have focused on private vehicles, typically assuming that vehicles are used once or twice a day and charged at home at night (Mukherjee and Gupta, 2015; Liu et al., 2015; Richardson, 2013). On the other hand, studies on shared autonomous vehicles have mainly dealt with the transport aspects, without considering charging optimization.

The difference in time scales for charging optimization and transport scheduling pose significant challenges for optimization of the system as a whole. While the rebalancing of vehicles is generally optimized with a horizon of 15-30 minutes (Zhang et al., 2016; Spieser et al., 2014), electric vehicle charging is typically optimized over several hours. The longer time frame for charging optimization is due to several reasons. Firstly, charging is relatively slow. Even with fast-charging it may take over one hour to fully charge a battery (Yilmaz and Krein, 2013). Charging for a relatively long time once a vehicle is connected is also more efficient, as it avoids the time wasted in continuous connection and disconnection. The most important consideration however is related to the challenges and opportunities that these vehicles offer to the grid as mentioned before. In order to avoid grid congestion and provide useful service to the grid, the charge scheduling algorithm needs to optimize over the time frame of variability of electricity demand and intermittent renewable energy sources such as wind and solar, which can be several hours.

In this work, charge optimization based on electricity price is integrated 
into model predictive control (MPC) of a shared autonomous electric vehicle (SAEV) system based on the work in Zhang et al. (2016). The novel model deals with the different time frames at which transport service and charging have to be optimized with a MPC routine which is run in parallel at two different time scales. Vehicle charging is optimized over longer time scales to minimize electricity costs. Vehicle routing and rebalancing for transport service is optimized at shorter time-scales to minimize waiting times for passengers, taking as constraints the results of the long-time-scale optimization. This approach allows the efficient optimization of both aspects of SAEV operation.

The work is organized as follows. In section 2 we present an overview of the related work. In section 3 we introduce the proposed methodology, including the transport model from literature, the charge optimization algorithm, and the two-layer model predictive control. In section 4 we apply the model to a case study in Tokyo and we discuss the results. This section also presents the methodology used for the transport demand generation from the survey and the electricity price scenarios reduction. In section 5 we present our conclusions and future work.

\subsection{Related work}

The literature on the topic of charging of electric vehicles is vast. Work has mainly dealt with the problem of optimal charging strategies, charging station deployment, and charging behavior analysis. Recent examples of literature on optimal location of public charging station in urban environments include He et al. (2015); Li et al. (2016); Dong et al. (2014); Chen et al. (2017). Tu et al. (2016) focuses on the optimization of charging station positioning 
for electric taxis. The problem of optimal siting of wireless charging facilities is also extensively studied with recent work including Riemann et al. (2015); Birrell et al. (2015). The optimization of charging station deployment was not considered in this work, and could be a focus of future research.

The problem of optimal charging strategies and the impact of electric vehicles on the grid is the main focus of this work, and has also been studied extensively in the literature, however mostly from the point of view of private vehicles. Waraich et al. (2013) investigates the impact of electric vehicle charging on the electricity grid with an agent-based approach, and find that smart charging schemes can overcome the problems associated with charging on the electricity distribution network. Marmaras et al. (2017) studies the effect of driver behavior on the transport and electric power system. The problem of individual charging behaviour and its impact on the power grid is also examined in Daina et al. (2017), where the response of drivers to electricity price signals is analyzed. This is in contrast to most previous studies that consider transport patterns as exogenous factors. $\mathrm{Hu}$ et al. (2016) present a review of smart charging literature for electric vehicle fleet operators. They find that centralized control has the best performance in the control of EV fleets and in the optimization of charging profiles in a smart grid. In this context, linear programming-based techniques are mostly used and offers the best performance. They also find it is essential to have a forecast of electricity price and EVs driving pattern. Mwasilu et al. (2014) review the vast literature on the interaction of electric vehicles with renewable energy sources.

While there is abundant literature on the topic of private electric vehicle 
charging, work dealing with the design of charging strategies and algorithms for shared electric vehicles is more limited. Freund et al. (2012) develop a control and optimization system for the charging of shared electric vehicles in a smart microgrid in order to maximize the use of renewable energy sources. Boyacı et al. (2015) introduce a model of an electric vehicle car sharing system with reservation. They consider charging stations serving requests in the vicinity. The optimization algorithm is also used to determine the optimal fleet size by maximizing the net revenues for the car sharing operator and the benefit for users, and taking into account the necessary car relocation among charging stations. Several authors explore the feasibility of taxi services using electric vehicles. Bischoff and Maciejewski (2014) simulate a fleet of electric non-autonomous taxis with MATSim, however without considering the implications for the grid. Wang and Cheu (2013) also investigate the operation of a electric taxi fleet with trip reservations, studying the case of Singapore. In these studies, dynamic electricity price-based smart charging is not considered.

The literature on the operation of shared autonomous electric vehicles is mostly focused on the transportation aspects of the problem, and in particular on the minimization of waiting times for passengers through the optimization of vehicle redistribution. In most cases the charging problem is not considered, presumably assuming non-electric vehicles (Fagnant and Kockelman, 2014; Hörl, 2016; Liu et al., 2017; Spieser et al., 2014; Levin et al., 2017; Pavone et al., 2012; Volkov et al., 2012; Acquaviva et al., 2014). Fagnant and Kockelman (2014) developed an agent-based model of shared autonomous vehicles using simplified transport assumptions. They used areas 
with homogeneous trip generation rates and a grid road network. They conclude that the system can provide adequate service with a fleet size of about a tenth of the equivalent number of private vehicles, with service quality dependent on user density. Spieser et al. (2014) used a more realistic transport model to study the minimum fleet sizing problem and estimate the economic impact of a fleet of shared autonomous vehicles replacing all other private transport modes in Singapore, based on real transport data. They find that the personal mobility needs of the entire population of Singapore can be met with a fleet size equal to a third of the total number of passenger vehicles currently in operation. Hörl (2016) predict the modal share of shared autonomous vehicles by developing an extension of MATSim. On a simplified road network, the results showed that the system could become the dominant transport mode, potentially disrupting public transport. Liu et al. (2018) propose a method to estimate the induced demand for shared autonomous transportation in a multimodal transporation system including public transport, based on the system's level of service. The framework also optimize the mobility on demand system characteristics taking into account policy decisions.

When electric charging of shared autonomous vehicles is considered, this is generally secondary to the transport optimization, and vehicles are assumed to charge as fast as possible independently of the grid conditions. Most studies agree that SAEVs can significantly reduce green-house gas (GHG) emissions and increase electrification in the transport sector (Greenblatt and Saxena, 2015; Weiss et al., 2017). Greenblatt and Saxena (2015) estimate that SAEVs could reduce GHG emissions by 87\%-94\% compared to current 
private vehicles in the United States. Even when accounting for a substantial increase in distance traveled due to higher convenience, SAEVs are still found to reduce emissions compared to the baseline scenario, mostly thanks to the fact that vehicle sizes can be optimized for each trip characteristics. A summary of selected works on SAEV that consider vehicle charging is given in Table 1, with more details below.

Chen et al. (2016) studied the operation of a SAEV system with a model based on Fagnant and Kockelman (2014). The agent-based transport model methodology is similar, but the investigation is expanded by including charging of the electric vehicles serving $10 \%$ of trip demand in a medium-sized metropolitan area. The analysis includes a charging station generation phase to find the number and position of charging stations needed to serve passengers within a certain waiting time. The model was run in different scenarios to investigate the sensitivity to several parameters. The study considers shortand long-range type of vehicles, with slow and fast charging. It was found that although double the number of vehicles are needed for the case with short-range and slow charging vehicles, this is the most profitable scenario. For the case study in Austin, Texas, the results indicate that each SAEV can replace between 5 and 9 private vehicles, depending on range and speed of charge. The model does not consider 'smart' charging and found that simultaneous charging of the fleet at peak times may be problematic for the electric grid. Farhan and Chen (2018) further expanded the model to incorporate ridesharing, showing that by allowing two passengers in the same vehicle, fleet size can be decreased to half and charging stations can also be significantly reduced. Allowing more than two passengers in a vehicle is 


\begin{tabular}{|c|c|c|c|c|c|c|c|}
\hline & $\begin{array}{l}\text { Opt. } \\
\text { rel. }\end{array}$ & Det. & Comments & $\begin{array}{l}\text { Realistic } \\
\text { road net. }\end{array}$ & C.S. & $\begin{array}{l}\text { Charging } \\
\text { stations }\end{array}$ & Formulation \\
\hline Bauer et al. (2018) & yes & yes & $\begin{array}{l}\text { Analysis of impact of dynamic } \\
\text { electricity prices wihtout change } \\
\text { in charging behavior. Detailed } \\
\text { cost analysis, emissions impact }\end{array}$ & $\begin{array}{l}\text { yes, with } \\
\text { traffic } \\
\text { conditions }\end{array}$ & no & $\begin{array}{l}\text { optimized } \\
\text { position } \\
\text { with } \\
\text { heuristics, } \\
\text { sensitivity } \\
\text { of number } \\
\text { of charging } \\
\text { stations }\end{array}$ & $\begin{array}{l}\text { agent- } \\
\text { based }\end{array}$ \\
\hline Biondi et al. (2016) & no & no & $\begin{array}{l}\text { Stochastic optimization of } \\
\text { charging station deployment } \\
\text { considering parking availability, } \\
\text { quantification of electric demand } \\
\text { (non-autonomous free floating car } \\
\text { sharing system) }\end{array}$ & $\begin{array}{l}\text { no, based } \\
\text { on cells }\end{array}$ & no & $\begin{array}{l}\text { yes, } \\
\text { optimized } \\
\text { positioning } \\
\text { and size }\end{array}$ & $\begin{array}{l}\text { set cover } \\
\text { problem }\end{array}$ \\
\hline Chen et al. (2018) & - & yes & $\begin{array}{l}\text { Studied the effect of different } \\
\text { pricing at charging stations. } \\
\text { Also considers load on electric } \\
\text { distribution networks }\end{array}$ & $\begin{array}{l}\text { simplified, } \\
\text { based on } \\
\text { graph }\end{array}$ & no & yes & MIQCP \\
\hline
\end{tabular}


with

Farhan and Chen (2018) yes

Iacobucci et al. (2018)

no



Kang et al. (2016)

Loeb et al. (2018) based on Chen et al. (2016), expanded with ridesharing

no

Trips generated stochastically, partial advance knowledge.

Considered connection time, minimum charging time.

Operating reserve potential estimation

Framework integrating 4 sub-problems: fleet size and assignment schedule; number and locations of charging stations; vehicle powertrain requirements; and service fees

\section{Tour-based transport model,} dynamic transport model with MATSim integration, sensitivity analysis no

no, based

on nodes

yes

heuris- position

agent-

based

nearest

neighbor

assignment

\section{$\begin{array}{ll}\text { tics) } & \text { with } \\ & \text { heuristics }\end{array}$ \\ $\begin{array}{ll}\text { tics) } & \text { with } \\ & \text { heuristics }\end{array}$}

$$
\text { assignment }
$$

\section{optimized}

positioning

nearest

neighbor

assignment no agent-

optimized based 


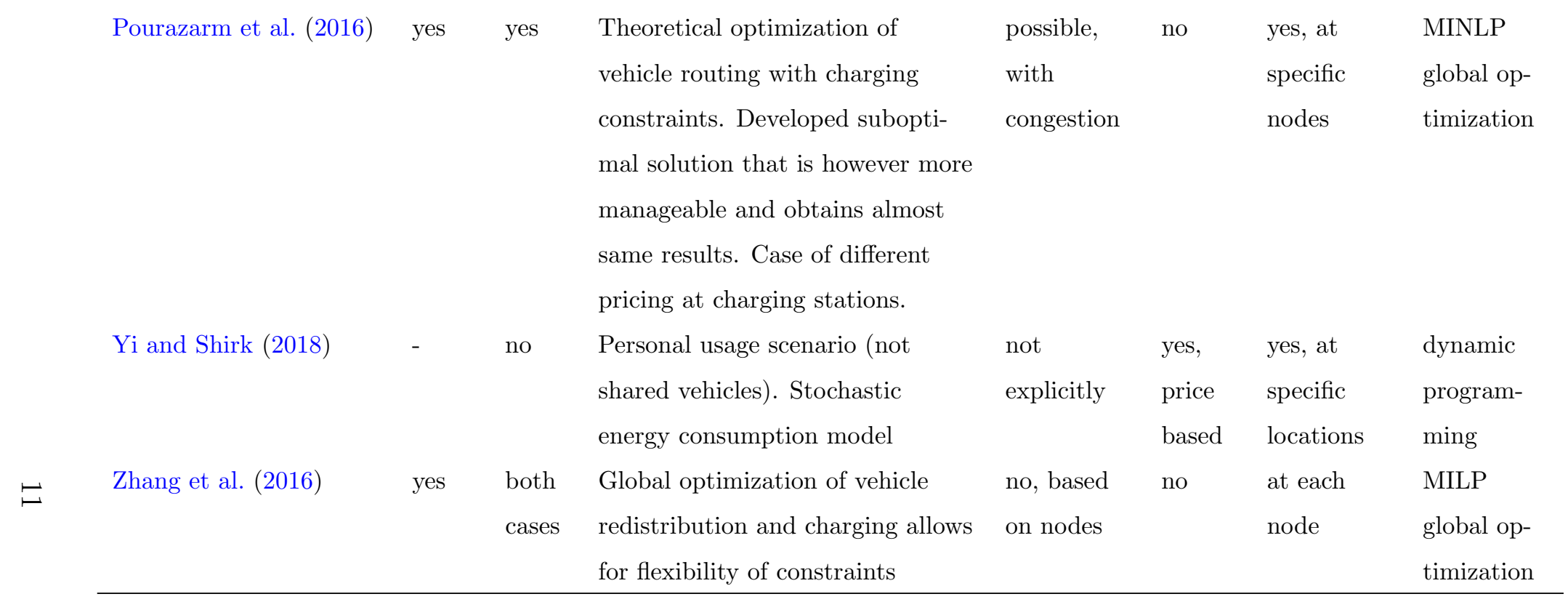

Table 1: Summary of the selected literature on SAEV modeling with focus on methodology. Only works dealing explicitly with charging during simulation time were considered. Opt. rel. = optimal relocation; Det. = deterministic model; C.S. = charge scheduling; MILP = Mixed-integer linear programming; MINLP = Mixed-integer non-linear programming; $M I Q C P=$ Mixed-integer quadratically-constrained programming 
shown to have rapidly diminishing returns, with marginal decrease in fleet size and a significant increase in waiting times.

Pourazarm et al. (2016) developed a theoretical optimization of vehicle routing with charging constraints. The problem is solved as a mixed integer nonlinear program (MINLP). The work also presents a faster problem with a sub-optimal solution that is however more computationally manageable and obtains almost the same results. The model allows for a variable electricity pricing at charging stations, however time-vaying pricing is not considered.

Chen et al. (2018) developed a framework for the optimal routing and charging of electric vehicles in a fleet context. The problem is formulated as a pick-up and delivery problem in a graph and is solved as a mixed-integer quadratically constrained program. The work also includes a discussion of the possible impacts of the system on the electricity distribution network. As in Pourazarm et al. (2016), charging optimization also considers different electricity pricing at stations but no time-varying electricity pricing is considered.

Iacobucci et al. (2018) developed a simulation model for SAEV in Tokyo with a heuristics-based charge scheduling and evaluate the potential for the system to decrease charging costs and provide operating reserves to the electric grid. They evaluate the system with a case study in Tokyo. The results show that SAEVs can effectively satisfy trip requests with a fleet of about 58 vehicles per thousand trips per day (1.4 vehicles per trip per hour) even when considering a very large area of $1600 \mathrm{~km}^{2}$. They are also found to be about 10 times cheaper than traditional taxis. The proposed heuristics charge scheduling is effective at lowering prices, although with a very variable 
generated price profile.

Bauer et al. (2018) developed an agent-based model for the simulation of $\mathrm{SAEV}$. The model proceeds through the day assigning trips to the nearest vehicle. If vehicles are not available within a threshold waiting time, a "new" vehicle is added to the necessary vehicles to satisfy demand. An iterative process was used to optimize the positioning of charging stations by starting with chargers everywhere and eliminating at each iteration the least used chargers. The model was tested in Manhattan with transport data from taxi trips. They find the optimal battery size and number of charging stations to minimize costs through sensitivity analysis. They conclude that vehicles with 50-90 miles range and 66 chargers per square mile (25 per square $\mathrm{km}$ ) with a $11 \mathrm{~kW}$ connection can provide service with a cost of $\$ 0.29-\$ 0.61$ per mile (\$0.18-\$0.38 per $\mathrm{km})$, about 10 times lower than normal taxis and lower than if the service was provided by any non-electric vehicle. They also calculate that SAEVs would reduce GHG emissions by $73 \%$ compared to current taxis with the current power grid thanks to higher vehicle efficiency.

Loeb et al. (2018) expand on previous work to develop a realistic framework for the simulation of SAEVs, especially for charging requirements. Sensitivity analysis was conducted to understand the influence of SAEV technology decisions on fleet operation. The authors investigates a case study in Austin, Texas. They found that the number of charging stations needed is highly dependent on vehicle range, but not on fleet size or charge times. Wait times are highly dependent on fleet size, or number of vehicles per passenger. Decreasing charge time has a rapid diminishing effect on wait times. Smart charging and the impact on the power grid were not considered. 
Zhang et al. (2016) present a model predictive control approach for the optimization of an autonomous car-sharing system with optimal rebalancing and consideration of vehicle charging constraints. The problem is solved as a mixed integer linear program (MILP). The algorithm is shown to outperform all the previous control strategies considered. The proposed optimization assumes uncontrolled charging of vehicles.

The literature on SAEVs almost completely avoids the problem of charge scheduling taking into account the electric power grid. As these systems are deployed at scale, however, the problem of optimal charging becomes apparent, as the increased load and charging peaks on the grid would be problematic if not managed (Waraich et al., 2013). Moreover, electric vehicles offer the opportunity for large scale demand response that can help increase the penetration of intermittent renewable energy in the grid (Dallinger and Wietschel, 2012).

\subsection{Contributions}

The objective of this research is to develop a framework for the optimization of vehicle fleet charging for shared autonomous electric vehicles taking into account real-time dynamic electricity price. While this problem has been considered extensively for private vehicles, it has been overlooked in the literature on SAEVs.

The problem of vehicle charge scheduling in smart grids with SAEVs is fundamentally different from the case of private vehicles. This is mainly due to different vehicle availability dynamics, which are due to the different times of connection to charging stations. Private vehicles would tend to be connected during the night, but are likely to be disconnected during the 
day as passengers stay at their destination. On the contrary, SAEVs can be connected at any time when they are not transporting passengers. Moreover, their connection can be controlled and planned. Another important difference is that aggregated charging dynamics are free from the constraints of individual vehicle charging, as passengers can use another vehicle on their return trip, for example. SAEVs charge scheduling also present the added complexity of the trade-off between charging cost minimization and wait times minimization, especially in the case of highly variable electricity price.

The aim of this work is to provide a multi-objective optimization algorithm for the simultaneous optimal redistribution and charging of SAEVs, taking into account both transport wait time minimization and charging cost minimization with dynamic time-varying electricity pricing. This work also consider realistic optimization horizons to account for limited advanced knowledge and two-layer optimization at different time scale to capture the different time scales inherent to vehicle redistribution and charge optimization. To the best of our knowledge, this is the first work to consider optimal relocation and charging with time-varying electricity price for SAEVs.

\section{A. State variables}

$d_{i, j}(t) \quad$ waiting passengers at node $\mathrm{i}$ with destination $\mathrm{j}$

$u_{i}^{k}(t) \quad$ vehicle $\mathrm{k}$ waiting at $\mathrm{i}$

${ }^{T} p_{i}^{k}(t) \quad$ moving vehicles

$q^{k}(t) \quad$ state of charge

\section{B. Control variables}

$v_{i, j}^{k}(t) \quad$ vehicle $\mathrm{k}$ transporting passengers between $\mathrm{i}$ and $\mathrm{j}$ 
$w_{i, j}^{k}(t) \quad$ vehicle $\mathrm{k}$ rebalancing empty between $\mathrm{i}$ and $\mathrm{j}$

$e^{k}(t) \quad$ energy charged

$g^{k}(t) \quad$ energy discharged with $\mathrm{V} 2 \mathrm{G}$

C. Exogenous simulation variables

$c_{i, j}(t) \quad$ passenger arrivals at node $\mathrm{i}$ with destination $\mathrm{j}$

$f(h) \quad$ relative number of trips departing at hour $h$

$m(t) \quad$ price of electricity

$p_{i, j}(t) \quad$ probability of a trip from $i$ to $j$ at time step $t$

$r w_{x}, r t_{x}, r o_{x}, r d_{x}$ weight, hour of departure, origin node, and destination node

of trip $x$ from survey.

$\lambda(t) \quad$ rate of trips arrival at time $t$

D. Parameters and constants

$J_{x}, J_{u}, J_{m}, J_{s} \quad$ cost of moving, rebalancing, charging, and final SOC

$q_{\min }, q_{\max } \quad$ minimum and maximum SOC

$t_{\text {hor }} \quad$ optimization horizon

$\alpha_{c}, \alpha_{v 2 g}, \alpha_{d} \quad$ charge, $\mathrm{V} 2 \mathrm{G}$ discharge, and consumption rate

$\beta$

temporal ratio between the 2 layers

$\rho_{1}, \rho_{2}, \rho_{3}$

weights of rebalancing, charging and final SOC secondary

objectives

$\eta$

V2G efficiency

E. Acronyms

MPC Model predictive control

SAEV Shared autonomous electric vehicle

SOC State of charge

V2G Vehicle to grid 
Table 2: Nomenclature

\section{Methodology}

The present work is based on the article by Zhang et al. Zhang et al. (2016) where an MPC was developed to find optimal management strategies for rebalancing of autonomous mobility-on-demand systems (shared autonomous vehicles). Although the work also proposed a version with charging constraints, the charging was not optimized and the vehicles would charge at maximum power as soon as they connect to charging stations. The rebalancing problem is formulated as a MILP. The problem formulation ensures that the system always optimizes the rebalancing of the vehicles within the optimization horizon. However, the computational complexity of the MILP optimization limits the feasibility to relatively small systems (in the order of a few tens of nodes). Nevertheless, the results of this model can be useful to estimate the performance in comparison to other systems. In this work, Zhang's model is extended to include electricity price-based charge scheduling and vehicle-to-grid discharge in the global optimization.

\subsection{Transport model from literature}

In this section we briefly report the original model from Zhang et al. (2016) for clarity. In the equations all the variables are considered normalized for simplicity. For example, charging power variables are power divided by battery capacity, and electricity price is price per fraction of battery capacity. This is to avoid including several multipliers (battery capacity, time step 
duration, etc.) and leave the equations clean. This does not affect the results as long as the parameters are normalized first.

The general formulation of the model evolution is:

$$
x(t+1)=A x(t)+B y(t)+c(t)
$$

where $x(t)$ is the state of the system at time $t, y(t)$ is the set of control variables and $c(t)$ represents new passenger arrivals at nodes.

$$
x(t)=\left[\begin{array}{l}
d(t) \\
p(t) \\
u(t) \\
q(t)
\end{array}\right] \quad(2) \quad y(t)=\left[\begin{array}{l}
v(t) \\
w(t) \\
e(t) \\
g(t)
\end{array}\right]
$$

These variables are described as follows.

The controls for the optimization are encoded into binary variables. $v_{i j}^{k}(t)=$ 1 when vehicle $k$ is transporting passengers between $i$ and $j$ and $w_{i j}^{k}(t)$ is the equivalent for rebalancing trips (empty trips). $d_{i j}(t)$ and $c_{i j}(t)$ are, respectively, the number of passengers waiting and arriving at node $i$ with destination $j$ at time $t . d_{i j}(t)$ evolves as:

$$
d_{i j}(t+1)=d_{i j}(t)+c_{i j}(t)-\sum_{k} v_{i j}^{k}(t)
$$

Another binary variable is used to keep track of vehicles in movement: ${ }^{T_{i}} p_{i}^{k}(t)=1$ when vehicle $k$ is at distance $T_{i}$ from its destination $i$ at time $t$. This allows the tracking of vehicles as they move to their destinations. When vehicle $k$ departs from node $j$ with destination node $i$ at time step $t$, ${ }^{t_{j i}-1} p_{i}^{k}(t+1)$ is set to one. In the following time step, ${ }^{t_{j i}-2} p_{i}^{k}(t+1)$ is set to one, and so on, until ${ }^{0} p_{i}^{k}(t+1)$ is set to one to indicate the vehicle has 
arrived to destination. The variable therefore evolves as:

$$
{ }^{T_{i}} p_{i}^{k}(t+1)
$$

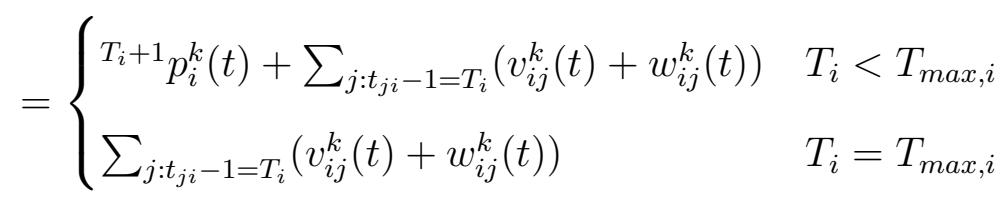

where $T_{\max , i}$ is the maximum distance in time steps between node $i$ and the other nodes. The binary variable $u_{i}^{k}(t)$ represents waiting vehicles at nodes. $u_{i}^{k}(t)=1$ when vehicle $k$ is waiting at node $i$ at time $t$. The variable evolves as:

$$
u_{i}^{k}(t+1)=u_{i}^{k}(t)+{ }^{0} p_{i}^{k}(t)-\sum_{k}\left(v_{i j}^{k}(t)+w_{i j}^{k}(t)\right)
$$

Vehicles can either be waiting or moving:

$$
\sum_{i} u_{i}^{k}(t)+\sum_{i, T_{i}}{ }^{T_{i}} p_{i}^{k}(t)=1
$$

Also, vehicles can only do one action at each time step:

$$
\sum_{i}\left(u_{i}^{k}(t)+\sum_{j} v_{i j}^{k}(t)+\sum_{j} w_{i j}^{k}(t)\right) \leq 1
$$

and vehicles cannot transport more passengers than are waiting at stations:

$$
\sum_{k} v_{i j}^{k}(t) \leq d_{i j}(t)+c_{i j}(t)
$$

Another constraint is associated with vehicles' charge state. The state of charge (SOC) of vehicles is encoded into a real variable $q^{k}(t) \in\{0,1\}$ and 
$q_{\min } \leq q^{k}(t) \leq q_{\max }$. Vehicles need to have enough charge to be assigned a trip:

$$
\begin{aligned}
& q^{k}(t) \geq v_{i j}^{k}(t) \alpha_{d} t_{i j}+q_{\min } \\
& q^{k}(t) \geq w_{i j}^{k}(t) \alpha_{d} t_{i j}+q_{m i n}
\end{aligned}
$$

$\alpha_{d}$ is the energy consumption per time step, and $t_{i j}$ is the number of time steps for the trip from $i$ to $j$.

The cost functions to minimize are related to the waiting time for passengers and the rebalancing costs:

$$
\begin{gathered}
J_{x}(x(t))=\sum_{i, j} d_{i j}(t) \\
J_{u}(y(t))=\sum_{k} \sum_{i, j} t_{i j} w_{i j}^{k}(t)
\end{gathered}
$$

For a more detailed description of the model, refer to Zhang et al. (2016).

As noted previously, in the original work the charging of vehicles was not a control variable, and vehicles would charge at a fixed rate when not moving until reaching a full state of charge or a movement was requested.

\subsection{Charge scheduling}

In this section and the next we report our extension to the model.

The decision on the charging of vehicles was added to the control vector, thus becoming part of the optimization. The state of charge (SOC) of vehicles $q^{k}(t)$ evolves as:

$$
q^{k}(t+1)=q^{k}(t)+e^{k}(t)-g^{k}(t)
$$


where $e^{k}(t)$ and $g^{k}(t)$ are, respectively, the energy charged and discharged to the grid in time step $t$, both non-negative.

The constraints for the charging rates are:

$$
\begin{aligned}
e^{k}(t) & \leq \alpha_{c} \sum_{i} u_{i}^{k} \\
g^{k}(t) & \leq \alpha_{v 2 g} \sum_{i} u_{i}^{k}
\end{aligned}
$$

where $\alpha_{c}$ and $\alpha_{v 2 g}$ are the maximum charging and V2G discharging rates, respectively. In this work we assume that vehicles can charge at any node as soon as they arrive. Moreover, we assume there are no congestion constraints at charging stations. Further constraints could be added to the model in the future.

To take into account the charging rate in the optimization, a further cost function is added. This is stated as:

$$
J_{m}(y(t))=\sum_{k \in V}\left(\left(e^{k}(t)-\eta g^{k}(t)\right) m(t)+\omega g^{k}(t)\right)
$$

where $m(t)$ is the price of electricity, $\eta$ is the V2G efficiency, and $\omega$ is the extra cost of cycling the battery incurred when supplying power to the grid. The system is considered a price taker, therefore the electricity price is not affected by charging. It should be noted that since $\eta<1$ and $\omega>0$, the optimization would never charge and discharge at the same time. Cost function $J_{m}$ is added as a further objective to the cost functions relative to the waiting time for passengers $J_{x}$ and the redistribution trips for vehicles $J_{u}$. 
Another cost function is added to put a premium on higher state of charge at the end of the optimization period:

$$
J_{s}=-\sum_{k \in V} q^{k}\left(t+t_{h o r}-1\right)
$$

This is to avoid vehicles reaching a minimum SOC at the end of the optimization horizon, and to account for future transport demand that is necessarily not represented in the optimization. Without this cost function, the optimization would tend to completely discharge vehicles at the end of the optimization period (horizon). The overall objective is therefore (the variables for each function have been omitted for compactness and clarity):

$$
\underset{y(t), . ., y\left(t+t_{\text {hor }}-1\right)}{\operatorname{minimize}} \sum_{t}\left(J_{x}+\rho_{1} J_{u}+\rho_{2} J_{m}\right)+\rho_{3} J_{s}
$$

$\rho_{1}, \rho_{2}$, and $\rho_{3}$ in (19) are the relative weights assigned to each secondary objective.

\subsection{Two-layer optimization}

As mentioned in the introduction, vehicles rebalancing and charging optimization require very different optimization time frames. Rebalancing is generally optimized within an horizon of half an hour or less, while it would be preferable to have a longer horizon for charging optimization in order to take into account the availability of intermittent renewable energy sources and variability of electricity demand.

In order to optimize both transport and charging, a two-layers model predictive control optimization was implemented. In model predictive control (or receding horizon control), at each time step the optimization is performed 


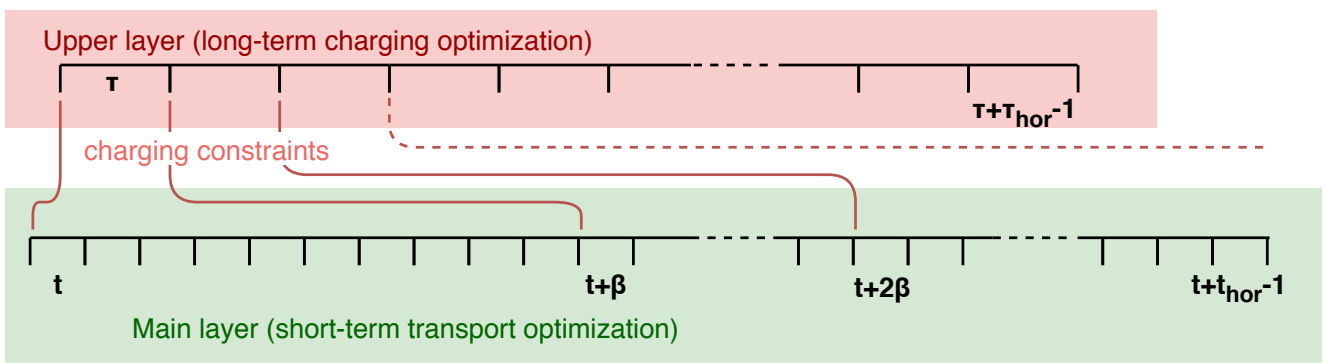

Figure 1: Schematic diagram of the two-layers MPC optimization model

over a time horizon and only the first control action is executed. This ensures that at each time step the control uses all the information available up to the future prediction horizon when taking the present action.

In this 2-layer MPC, a higher 'coarse' MPC layer optimizes for charging over longer time frames taking into account the requirements of transport service. The main layer optimizes transport service over short time frames, taking as constraints the optimal charging controls found by the higher layer. Both layers are based on the model presented in the previous sections.

The two layers are related by a step length ratio $\beta$ that determines the relative length of a time step between the two layers. At the beginning of the simulation, the higher coarse layer determines the optimal charging schedule by optimizing over its own time frame. The results are passed down to the main layer as constraints on charging during each step. After $\beta$ time steps in the main layer, the higher layer optimizes again over its own time frame, passing down the next charging constraints to the main layer for the next $\beta$ time steps. 


\subsection{Upper layer and constraint assignment}

In this section, the integration of the two layers is discussed. The upper layer simulation variables are denoted with Greek letters, so that $d, p, u, q$, $t, e, c$ are respectively $\delta, \pi, \mu, \phi, \tau, \epsilon, \kappa$. We also define $T_{i}^{k}$ as the distance from node $i$ of vehicle $k$ if moving, and $T_{i}^{k}=0$ if the vehicle is waiting at node $i$. The upper layer's objective function has secondary weights that are related to the main one by division by a factor $\beta$, to reflect the fact that a waiting passenger waits $\beta$ times longer in the upper simulation for each time step, as the time step is longer. The arrivals at nodes is defined as:

$$
\kappa_{i j}(\tau)=\sum_{t=(\tau-1) \beta+1}^{\tau \beta} c_{i j}(t)
$$

At the start of an upper layer simulation at (main) time $t^{\prime}$, we assign the main layer situation to the upper layer:

$$
\begin{gathered}
\delta_{i j}(\tau)=d_{i j}\left(t^{\prime}\right) \\
{ }^{\left[T_{i}^{k} / \beta\right]} \pi_{i}^{k}(\tau)=1 \quad \text { if } \quad T_{i}^{k}>0 \\
\mu_{i}^{k}(\tau)=u_{i}^{k}\left(t^{\prime}\right) \\
\phi^{k}(\tau)=q^{k}\left(t^{\prime}\right)
\end{gathered}
$$

The charging controls resulting from the upper layer optimization are assigned back to the main layer as constraints on charging and on movement. For vehicles moving, the constraints are assigned only after arrival. 


$$
\begin{gathered}
e^{k}(t)= \begin{cases}\epsilon^{k}\left(\tau^{\prime}\right) / \beta & t>t^{\prime}+T_{i}^{k} \\
0 & t \leq t^{\prime}+T_{i}^{k}\end{cases} \\
u_{i}^{k}(t)= \begin{cases}1 & t>t^{\prime}+T_{i}^{k} \\
0 & t \leq t^{\prime}+T_{i}^{k}\end{cases}
\end{gathered}
$$

where $\tau^{\prime}$ in $(25)$ such that $(\tau-1) \beta<t^{\prime}-t \leq \tau \beta$.

\subsection{Limitations of the model}

The model is based on a global optimization problem, and is therefore inevitably limited in scale. The computational complexity of the problem grows more than linearly with the size of the simulation (number of vehicles, number of nodes, time resolution etc.) becoming infeasible for more than a few tens of vehicles or for long optimization horizons. Compared to the original model, the addition of charge scheduling further limits the feasible scale since it adds further complexity and increases the number of variables. This is not a limitation of the SAEV system in itself, but rather of the global optimization approach. In this work, the two layer approach allows to have a longer optimization horizon with a trade-off with time resolution in the upper layer, while preserving a high resolution main layer with a shorter optimization horizon. For larger systems, such as practical implementations with thousands of vehicles, a faster and more scalable heuristics-based optimization model is needed. However, this model can be used as benchmark for future more practical heuristics-based models. 


\section{Numerical simulations}

The model was implemented in MATLAB and solved as a mixed integer linear optimization with the built-in MATLAB function intlinprog. To evaluate the performance of the model, several simulations were conducted using historical data from a transport survey in Tokyo.

\subsection{Transport data}

The Tokyo Person Trip Survey 2008 (Ministry of Land, Infrastructure, Transport and Tourism, Japan (MLIT), 2008) is a survey comprising around 2 million trips in the Tokyo metropolitan area. The 2008 survey is the latest available survey released for Tokyo. Although the survey is 10 years old, the demographics and infrastructure of Tokyo has remained stable and it is expected that this implies a relatively stable demand pattern when compared to 2008. Infrastructure and ridership of all major railways are mostly unchanged between 2008 and 2015, and in the same period, the length of roads in Tokyo city changed by less than 1\% (Tokyo Metropolitan Government, 2016).

Only trips by car or taxi with origin and destination in a central $5 \times 5$ $\mathrm{km}$ area of Tokyo were considered. These were considered the trips with characteristics more likely to be similar to trips done with the SAEV system. This area was divided into 10 regions by grouping nodes with k-means clustering (Fig. 2). The cluster's centroid for each area was considered as a discretized model of the origin/destination of trips in each area.A Poisson process was used to generate the stochastic arrivals at each time step, with frequency for each origin and destination nodes pair selected according to 
the survey. More information about the survey extraction methodology can be found in Iacobucci et al. (2018).

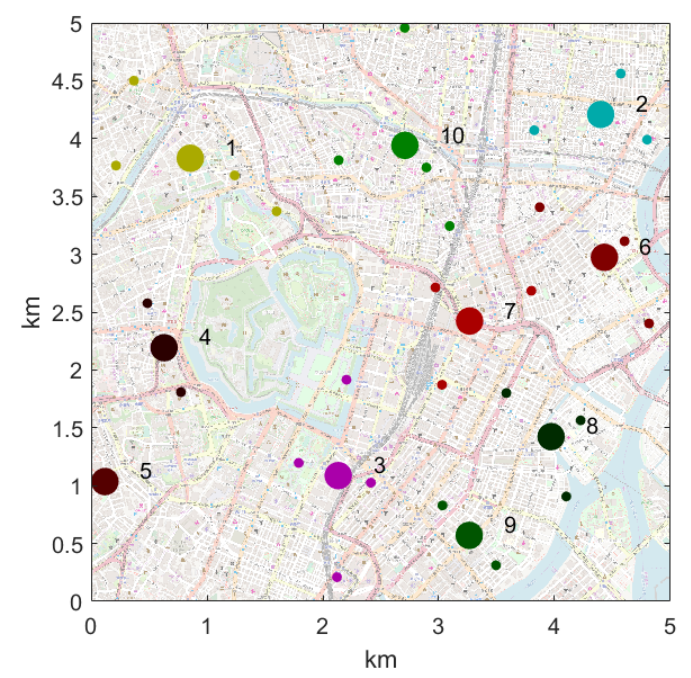

Figure 2: Map of the central $5 x 5 \mathrm{~km}$ area of Tokyo used in the simulations. Smaller circles represent origin/destination nodes in the survey, while larger circles represent the cluster centroids.

\subsection{Electricity prices and scenario reduction}

In order to investigate the influence of different electricity price profiles on the results, real electricity market price profiles were used in the simulations. Due to the computational time required for each simulation, studying an entire year of electricity prices would be extremely time consuming. A scenario reduction approach was therefore used to reduce the number of required simulations while assessing the behavior of the model in all the possible situations. 


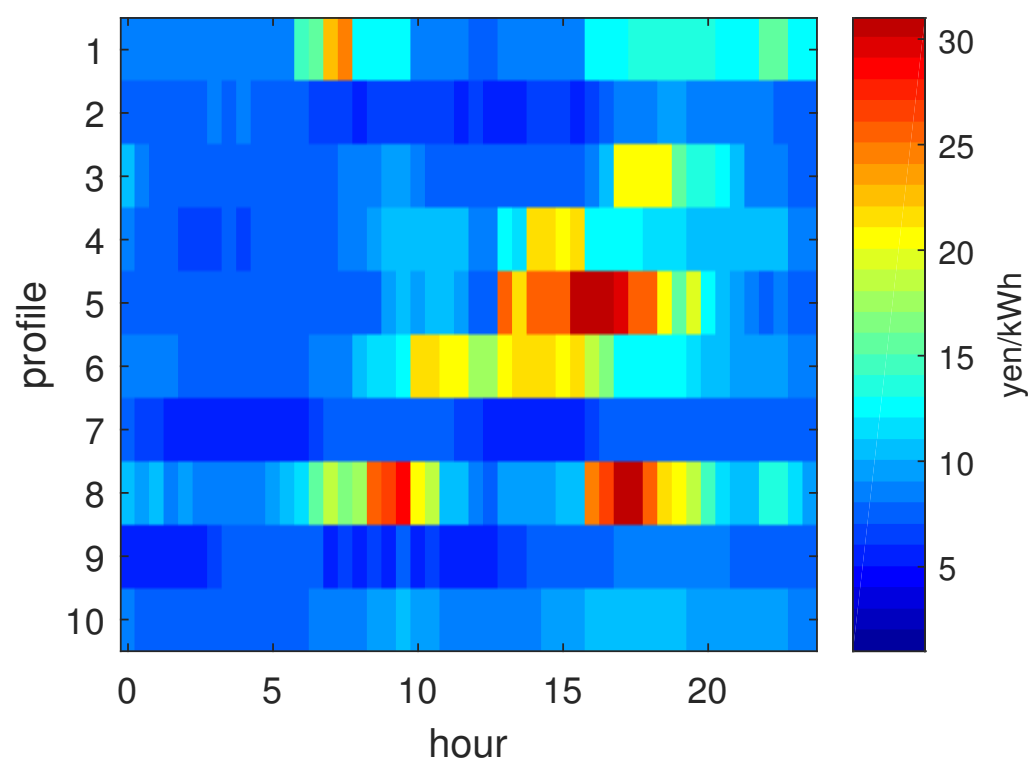

Figure 3: Day-ahead price profiles for the Tokyo region in the JEPX wholesale electricity market selected with scenario reduction.

In this work, a scenario is defined as the electricity price profile during a single day. In the scenario reduction approach a subset of all possible scenario (daily price profiles) is selected to represent the entire spectrum of possible scenarios, i.e., all the historical daily electricity profiles. Only the most representative scenarios are kept, and similar scenarios are discarded. The probability of the selected scenarios is updated with the sum of the probability of all the discarded scenarios most similar to the ones kept. To assess the similarity of different price profiles, the Kantorovich distance was used, which is the most common measure of distance between probability distribution used in stochastic programming (Conejo et al., 2010). All the price profiles were normalized to calculate the distance. 


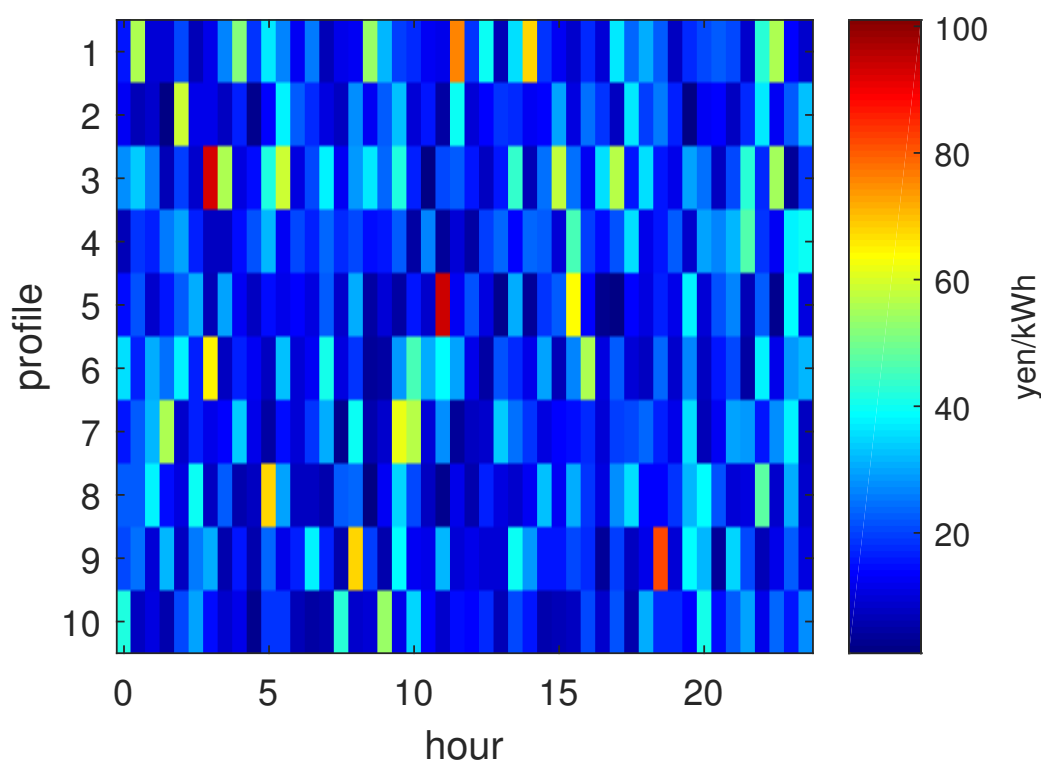

Figure 4: Generated scenarios from a gamma distribution to test the model with high variability prices.

The forward selection algorithm described in Conejo et al. (2010) was used to select the scenarios. The day-ahead wholesale electricity market prices for the Tokyo region on the Japanese Electric Power Exchange (JEPX) in financial year 2016 (April 2016 to March 2017) were used (JEPX, 2017). 10 scenarios were selected (Fig. 3). Each scenario was associated with a weight (probability) proportional to the number of days with a profile closest to the selected one (Table 3).

The Tokyo region has relatively low penetration of renewable electricity, and the wholesale market is still relatively small as the electricity market is still in the process of being completely liberalized. In order to test the model with price profiles with higher variability, 10 more scenarios were generated 
from a gamma distribution with shape parameter 2 and scale parameter 10, giving an average price of 20 yen/kWh (Fig. 4).

\begin{tabular}{c|c|c|c|c|c|c|c|c|c|c}
\hline & 1 & 2 & 3 & 4 & 5 & 6 & 7 & 8 & 9 & 10 \\
\hline JEPX & 0.6795 & 0.0110 & 0.0356 & 0.1205 & 0.0192 & 0.0082 & 0.0356 & 0.0055 & 0.0466 & 0.0384 \\
\hline Gamma (random) & 0.1 & 0.1 & 0.1 & 0.1 & 0.1 & 0.1 & 0.1 & 0.1 & 0.1 & 0.1 \\
\hline
\end{tabular}

Table 3: Weight (probability) of each scenario
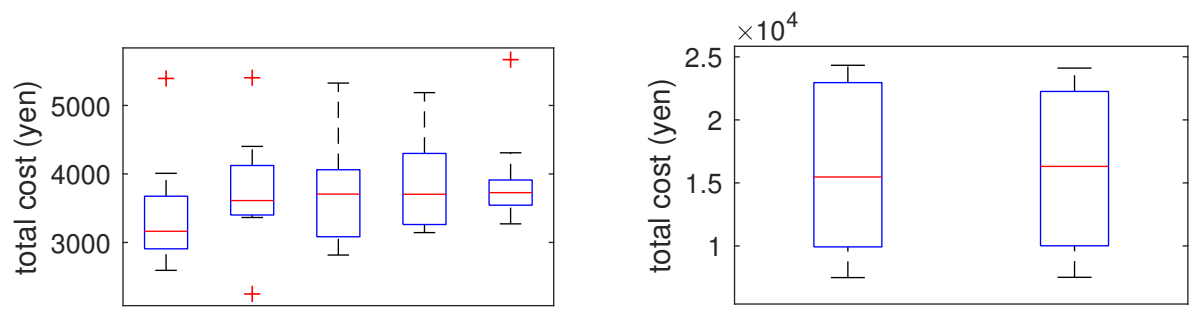

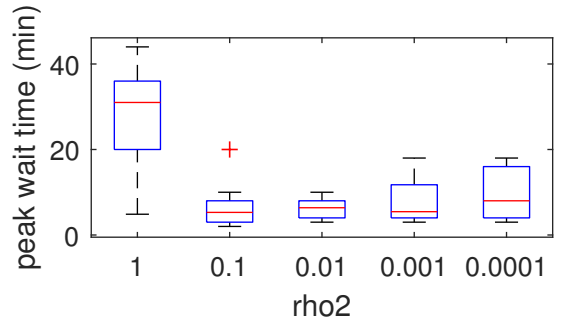

(a)

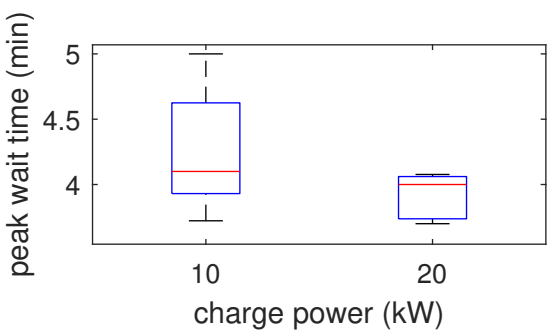

(b)

Figure 5: Costs and moving average (MA) peak waiting times sensitivity to: (a) secondary weight $\rho_{2}$ (b) charge power

\subsection{Secondary weights determination}

Deciding the value of secondary weights is an important problem for multi-objective optimization models. The first weight in (19), $\rho_{1}$, is the easiest to set, as there is an optimal rebalancing that can always be achieved. 
If set low enough (0.01 in this work), the rebalancing will be optimized without influencing the main objective. Further decrease of rebalancing cost is in theory impossible under this optimized rebalancing cost, equivalent to the Earth Mover's distance Spieser et al. (2014). Determination of $\rho_{2}$ and $\rho_{3}$ is less straightforward. These two secondary objectives represent the cost of energy, not the 'waiting cost' like the main objective. Therefore, setting these objectives depend on the relative importance of minimizing monetary costs over minimizing the waiting times for passengers. This trade-off needs to be decided by policy or by user preference.

A sensitivity analysis was conducted to understand the influence of this parameter on the cost and wait times. The results are shown in Figure 5a. Each value of $\rho_{2}$ was tested with 10 different price profiles, with a scaled down simulation with only 10 vehicles. Total system costs increase and waiting time decrease with decreasing $\rho_{2}$. However, a plateau is reached for both values, as there is a charge schedule that ensures minimum waiting time while minimizing as much as possible the charging costs. This is reached for $\rho_{2} \simeq 0.1$ in the simulations of Figure $5 \mathrm{a}$. In this work, $\rho_{2}$ was set to 0.001 to ensure the simulations are within the plateau region, as minimizing wait times is considered a priority over marginally lower charging costs. The objective for final SOC was set as $\rho_{3}=\rho_{2} m_{\text {median }}$ where $m_{\text {median }}$ is the median price of electricity. This is equivalent to compensating lower SOC by buying electricity at the median price up to $100 \%$ SOC.

\subsection{Sensitivity analysis}

A sensitivity analysis was performed on the full model to investigate the influence of some key parameters on the results. Each parameter was tested 

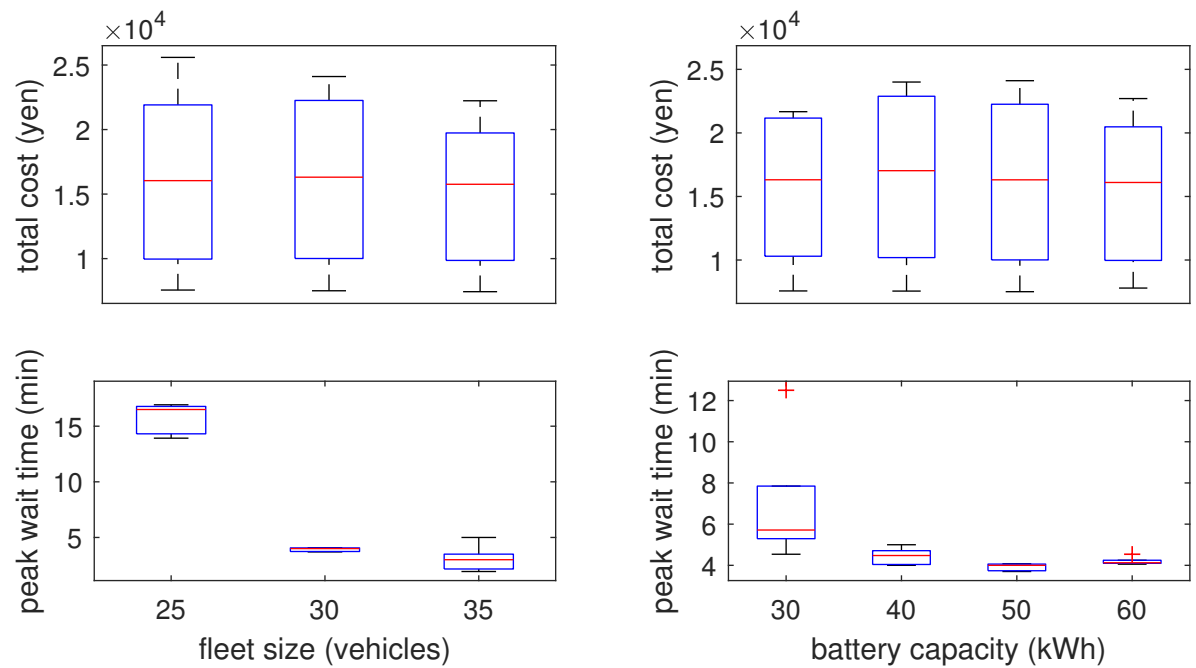

(a)

(b)
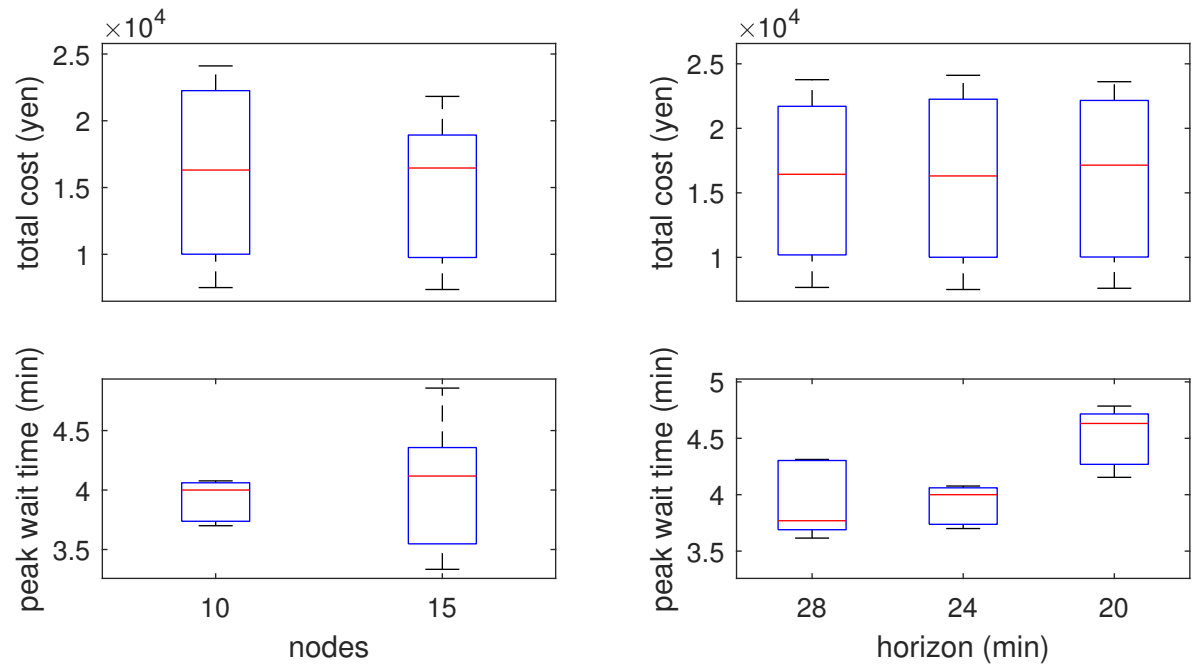

(c)

(d)

Figure 6: Sensitivity of 10-minutes moving average peak wait times and total costs for different parameters: (a) fleet size; (b) battery capacity; (c) number of nodes; (d) main layer optimization horizon. 


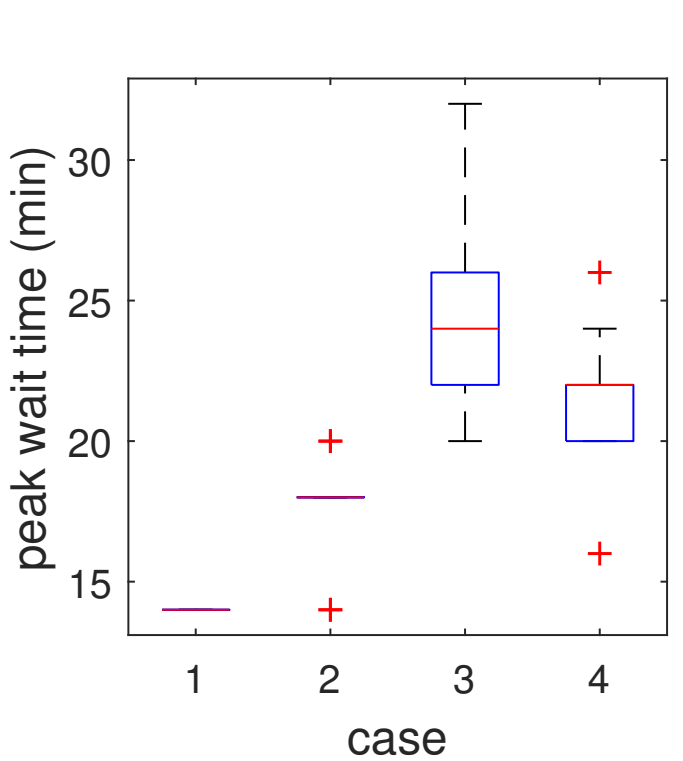

(a)

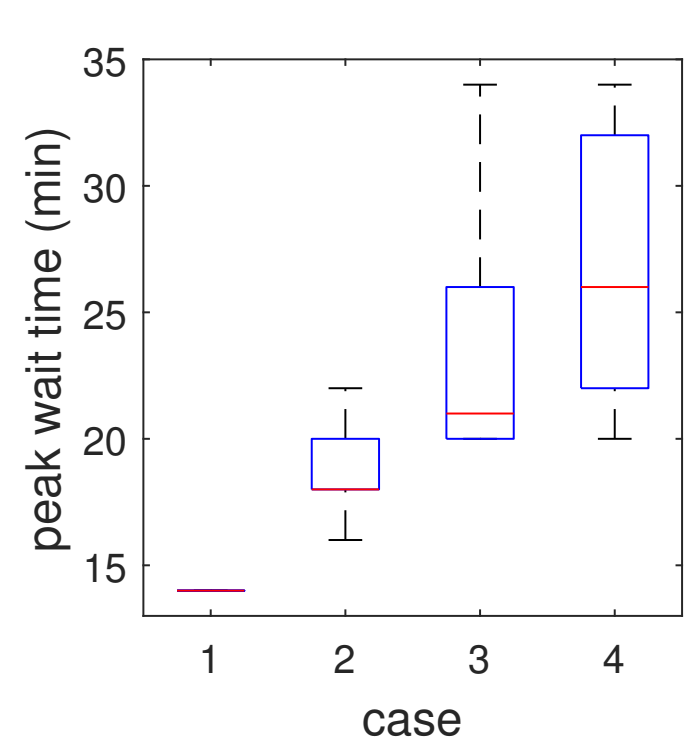

(c)

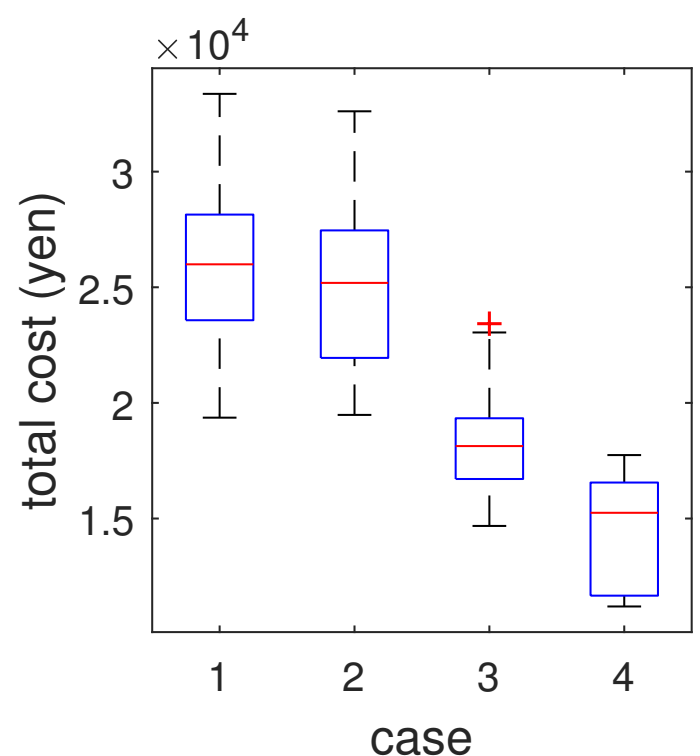

(b)

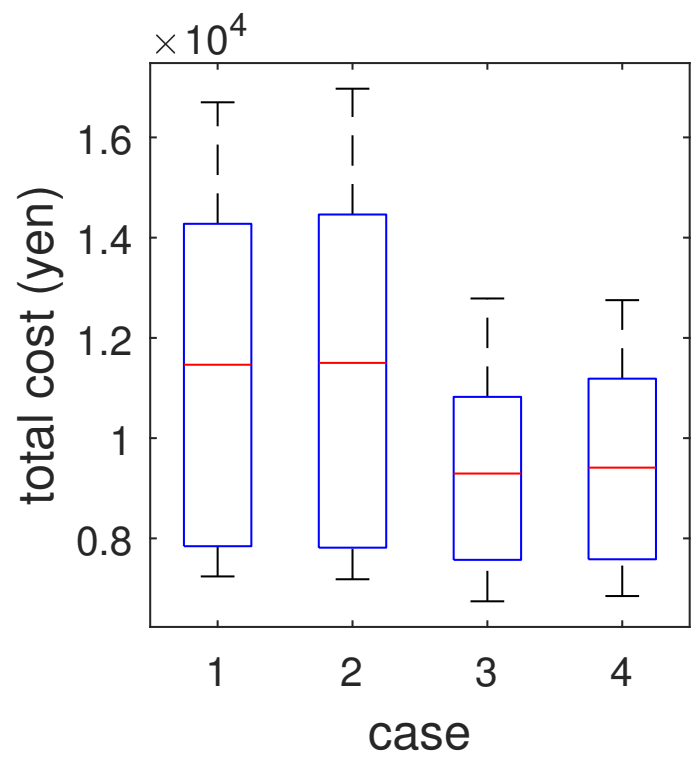

(d)

Figure 7: Peak wait times and total costs (a-b) for the random price profiles generated from a Gamma distribution; (c-d) for the simulation with real prices on the Japan Power exchange in 2017. The 4 cases represent the unscheduled model, the 1-layer model, the 2-layer model, an33 the 2-layer with V2G, respectively. 


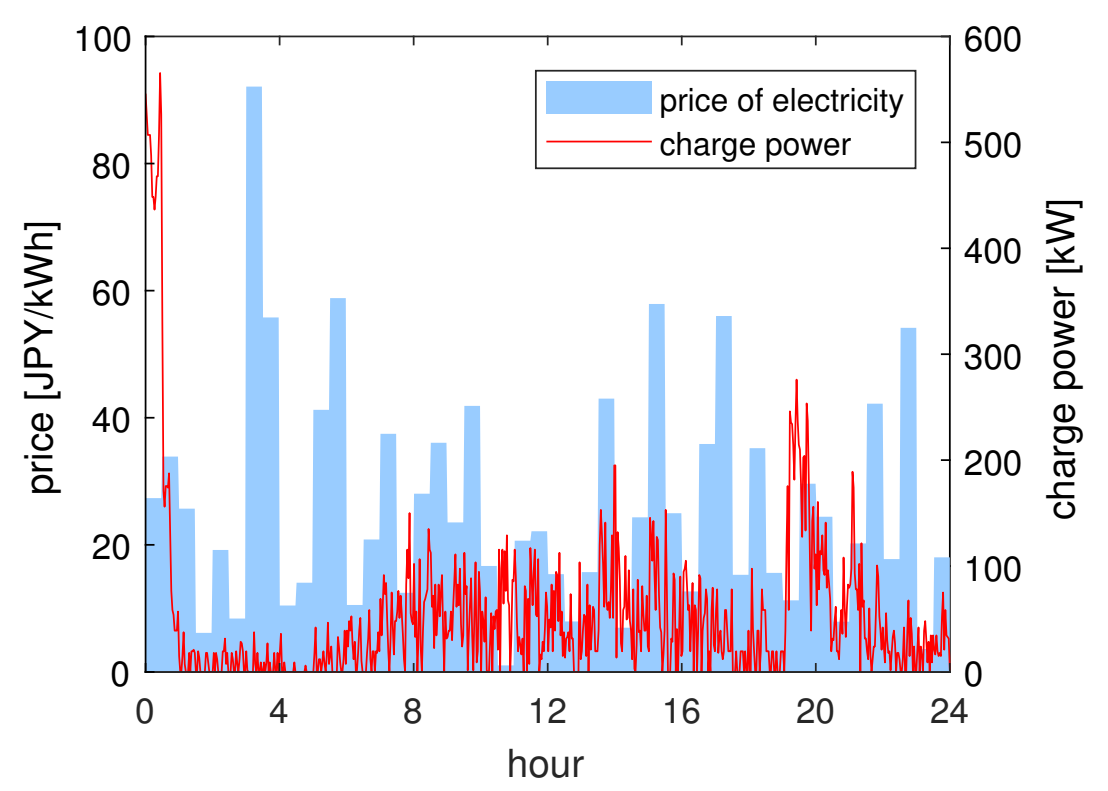

Figure 8: Aggregated charging power and electricity price for case 1 (unscheduled). The price profile is taken from a Gamma distribution.

on 5 different price profiles with the 2-layer model without V2G. The effect of two different charge power connections is reported in Fig. 5b. Faster connections decrease peak wait times, but has no appreciable effects on charging costs. This suggests that the total cost in these cases is not limited by charging speed, but by the need to have available and charged vehicles at specific times. It was also found that a lower charging speed would not allow the system to be stable, as vehicles would not be able to charge fast enough to satisfy all passenger requests without increasing the fleet size. Larger fleet sizes decrease peak wait times, as expected, up to a certain threshold. This is found to be about 30 vehicles for the amount of passenger requests considered (Fig. 6a). The effect is similar with increasing battery capacity 


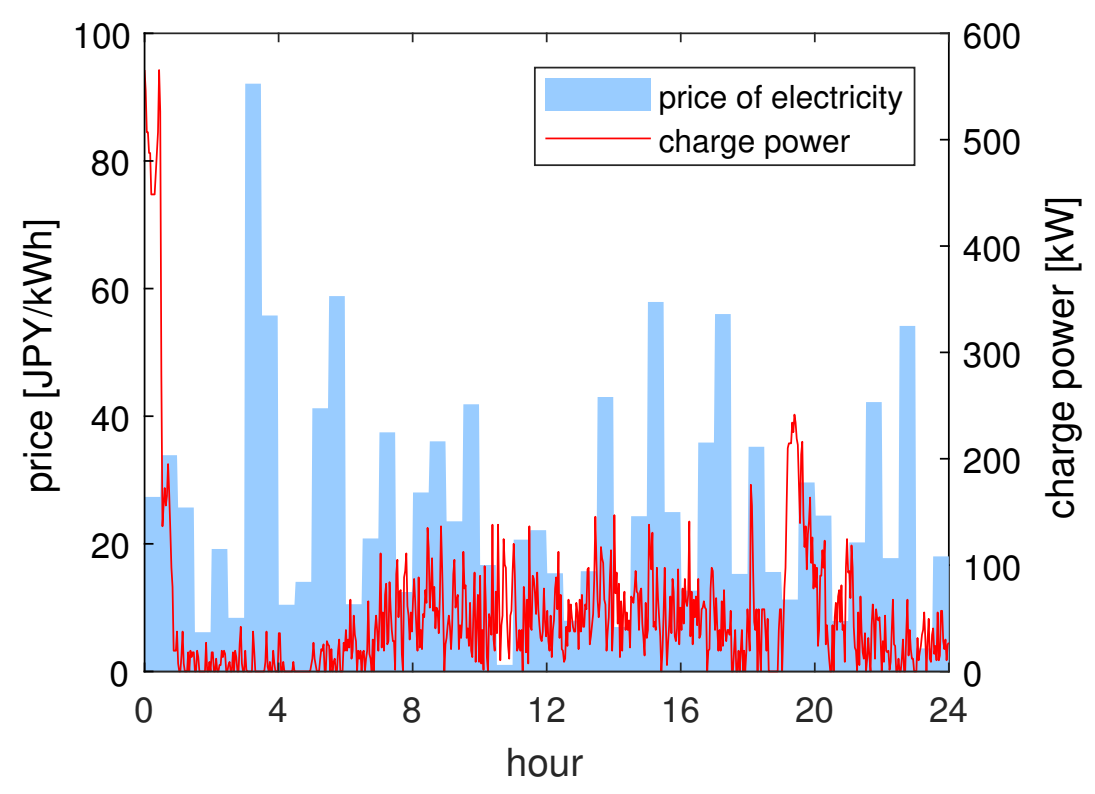

Figure 9: Aggregated charging power and electricity price for case 2 (1-layer scheduling). There is almost no difference between case 1 and 2, as the charge scheduling optimization horizon is too short to be effective.

(Fig. 6c). With a capacity of more than $50 \mathrm{kWh}$ there are no significant changes in peak wait times. It should be noted that total cost only refers to charging costs, and capital costs are not considered in this work. Larger fleets or larger battery capacities are inevitably linked to higher capital costs which would increase system cost significantly.

The number of nodes increases the complexity and realism of the simulation, thus increasing the variability across scenarios and the simulation time. However, median costs and median peak wait times are similar between 10 and 15 nodes (Fig. 6b). Longer optimization horizons in the main layer translate into lower peak waiting times, although the change is minimal after 
about 24 minutes (Fig. 6d). With horizons of less than about 20 minutes, the optimization fails as too many passenger requests accumulate due to sub-optimal routing, and request rejection was not included in the model.

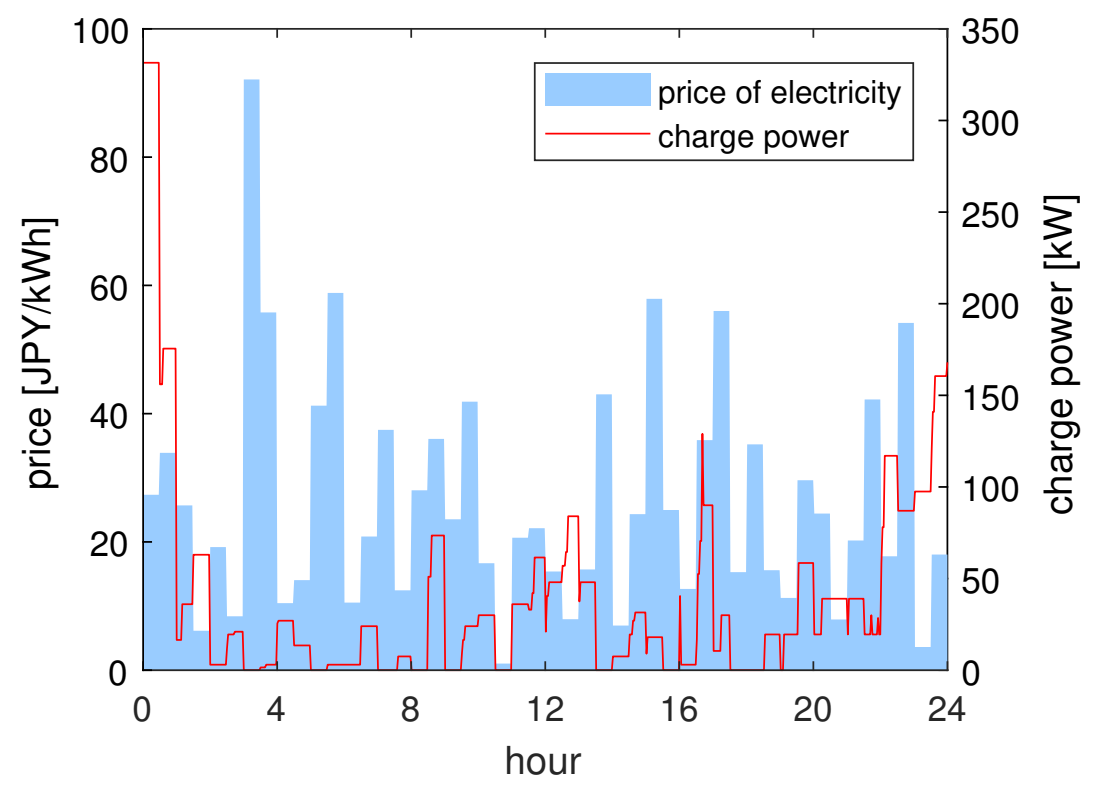

Figure 10: Aggregated charging power and electricity price for case 3 (2-layer scheduling)

\subsection{Results}

The simulations were run with 30 vehicles, and an average trip rate of 1500 trips per day. The speed of vehicles was set at $20 \mathrm{~km} / \mathrm{h}$, the reported average road speed in central Tokyo (Yabe, 2015).

Time step for the main layer is 2 minutes, with a 15 time steps horizon (30 minutes). The ratio between the two layers is $\beta=15$, so that the higher layer has a time step of 30 minutes. The time step horizon for the higher 


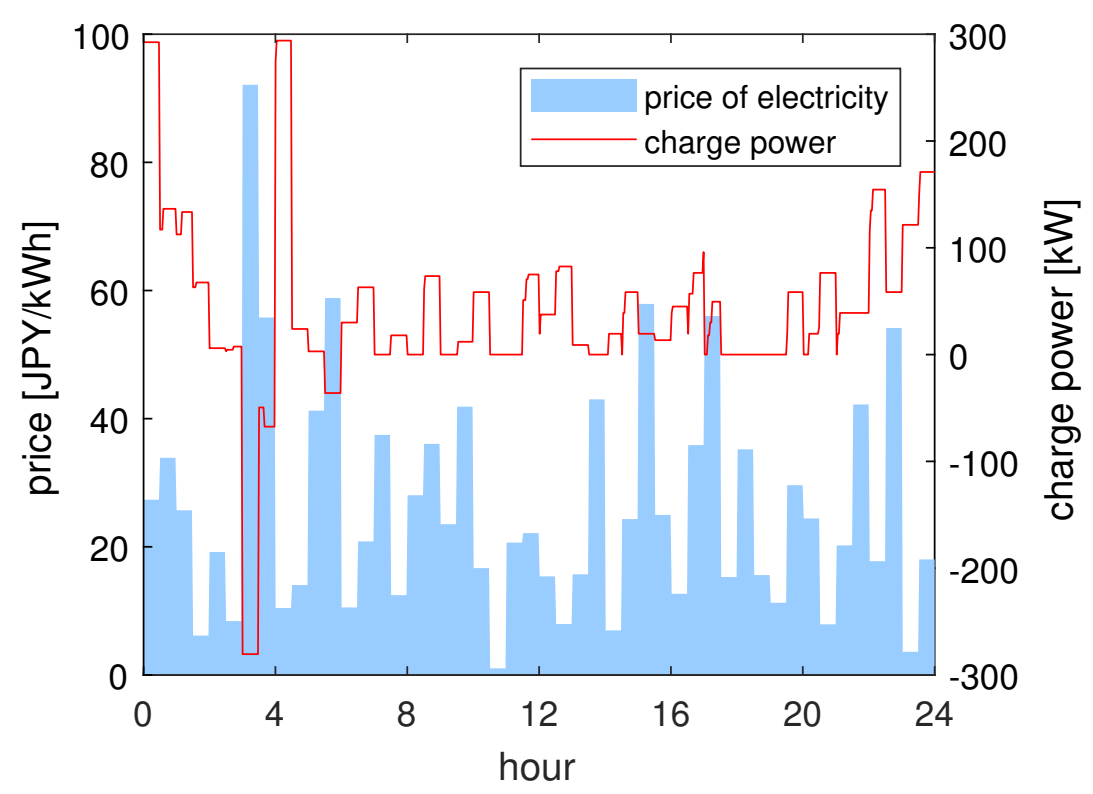

Figure 11: Aggregated charging power and electricity price for case 4 (2-layer scheduling with V2G)

layer is set at 10, giving an optimization time horizon for charging of 5 hours. Vehicles battery size was set at $50 \mathrm{kWh}$ and the state of charge was limited between 0.2 and 0.9 to increase battery life. The maximum power connection was set at $20 \mathrm{~kW}$ both for charging and V2G discharging. The initial SOC at the beginning of each simulation was set at 0.7 for all vehicles. This was chosen as a reasonable estimation of the average state of charge of vehicles at midnight of an average day. As the amount of passenger requests between midnight and about 6-7 am is generally very low, the SOC level at that time does not practically influence results in terms of transport quality, as vehicles have enough time to charge during the night if needed. The same SOC was used to be able to better compare results across simulations. 


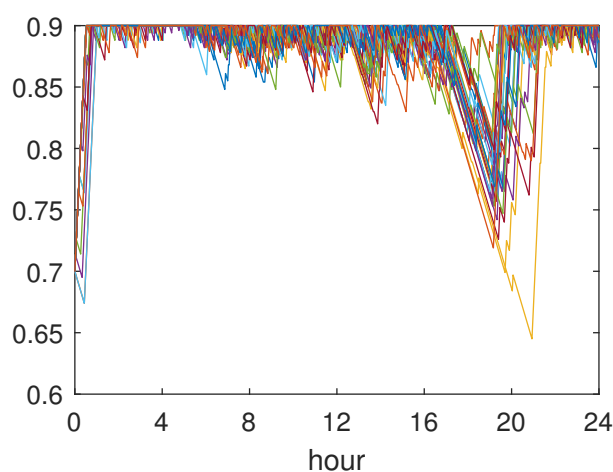

(a)

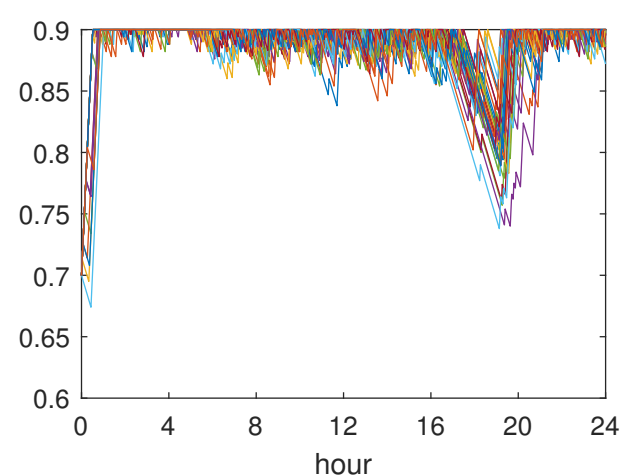

(b)

Figure 12: State of charge (SOC) of vehicles (a) for case 1 (unscheduled charging); (b) for case 2 (1-layer scheduling). In case 2, the scheduling does not have a significant effect due to the limited optimization horizon.

Different models were compared to evaluate the performance of the proposed charge scheduling algorithm in terms of waiting times and charging costs:

1. no charge scheduling

2. 1-layer charge scheduling

3. 2-layer charge scheduling

4. 2-layer charge scheduling with V2G

Different results may have different levels of SOC at the end of the simulation. To be able to compare different results the difference between the final SOC and the initial SOC was valued at the median price of the day.

The peak waiting times and the total costs for the simulations are presented in Figs. 7 for the case with random price profiles and with price profiles 


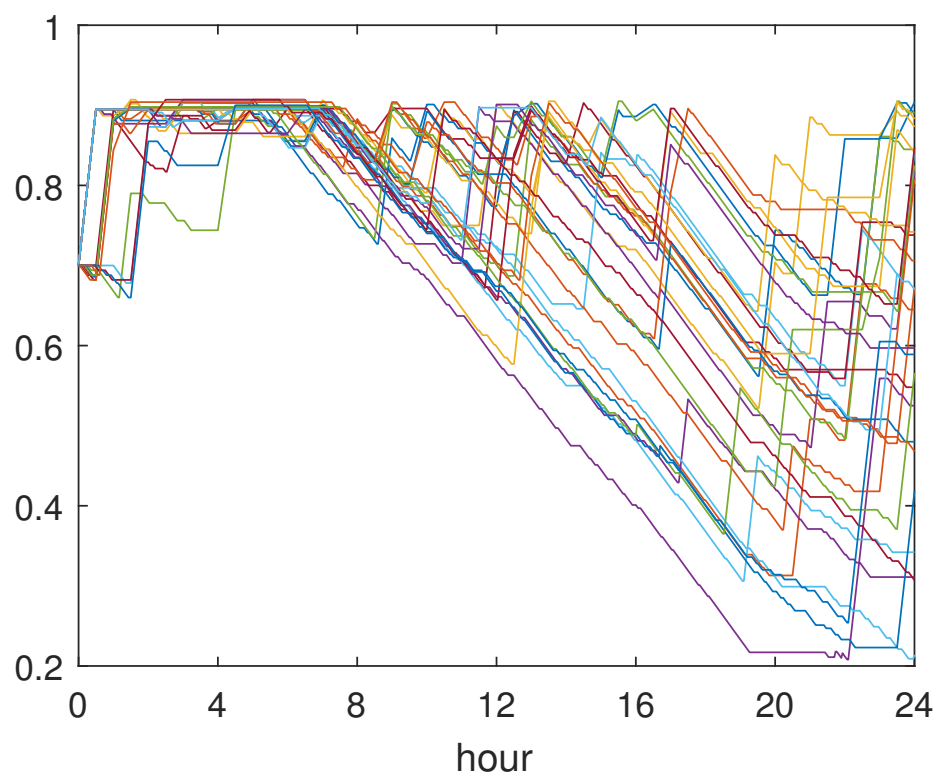

Figure 13: State of charge (SOC) of vehicles for case 3 (2-layer scheduling)

taken from JEPX. The model proposed with 2-layer optimization decreases total charging costs significantly when compared to the unscheduled model or the 1-layer model. The effect is much larger with the random price profile, due to the much higher variability that gives the charge optimized model a clearer advantage over the unscheduled model.

For the case with historical price profiles in Tokyo, the effect is smaller. As mentioned previously, this is due to the relatively low penetration of renewable energy sources in the Tokyo grid and the nascent state of the Japan wholesale electricity market, with both factors contributing to relatively static price profiles. Moreover, when peaks exist, they generally correspond to transport demand peaks (Fig. 3), rendering the model less effective since transport is always a priority in the optimization. Table 4 reports the results 


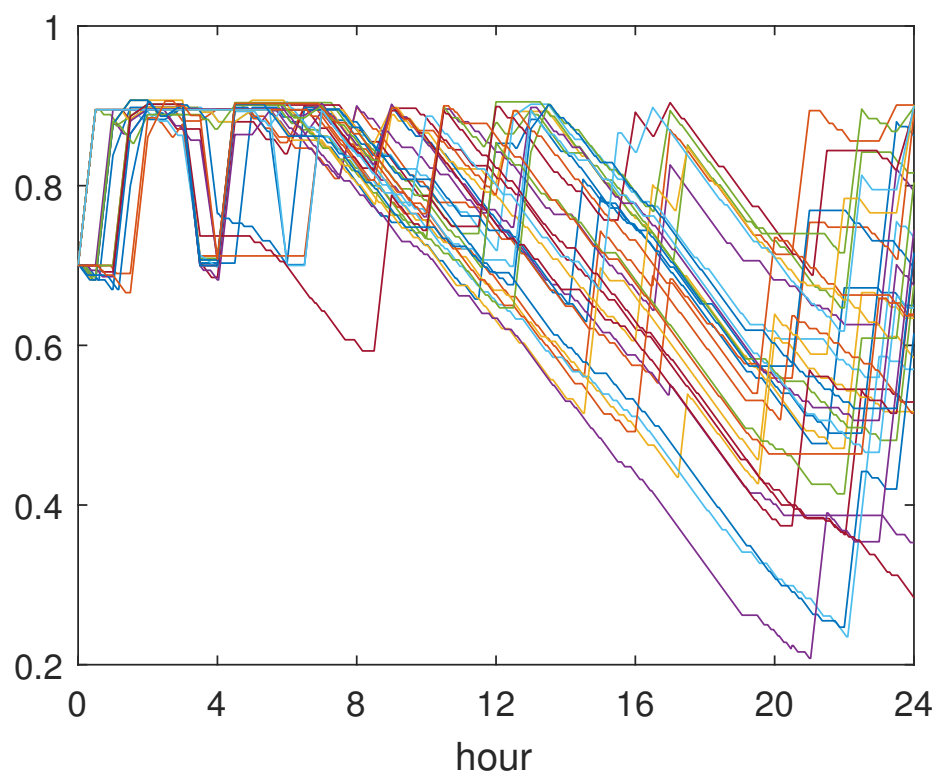

Figure 14: State of charge (SOC) of vehicles for the case with V2G. The steep drop in SOC around 4 am is correlated with a high electricity price and resulting V2G discharge (compare with Fig. 11).

for the JEPX prices weighted with the probability of each scenario tested. Costs decrease by about $10 \%$ with the proposed model.

While the model with V2G has a significant advantage over the one without in the high variability random profiles (Fig. 7b), the results for the two models are practically indistinguishable for the JEPX profiles (see Fig. $7 d$ and table 4). This is due to the relatively low price differentials in the JEPX profiles, which are generally not enough to overcome the costs of V2G in terms of battery degradation and opportunity cost. The higher variation of price profiles also creates unexpected results, such as that in Fig. 7a. Although it is reasonable to assume that V2G would generally tend to 


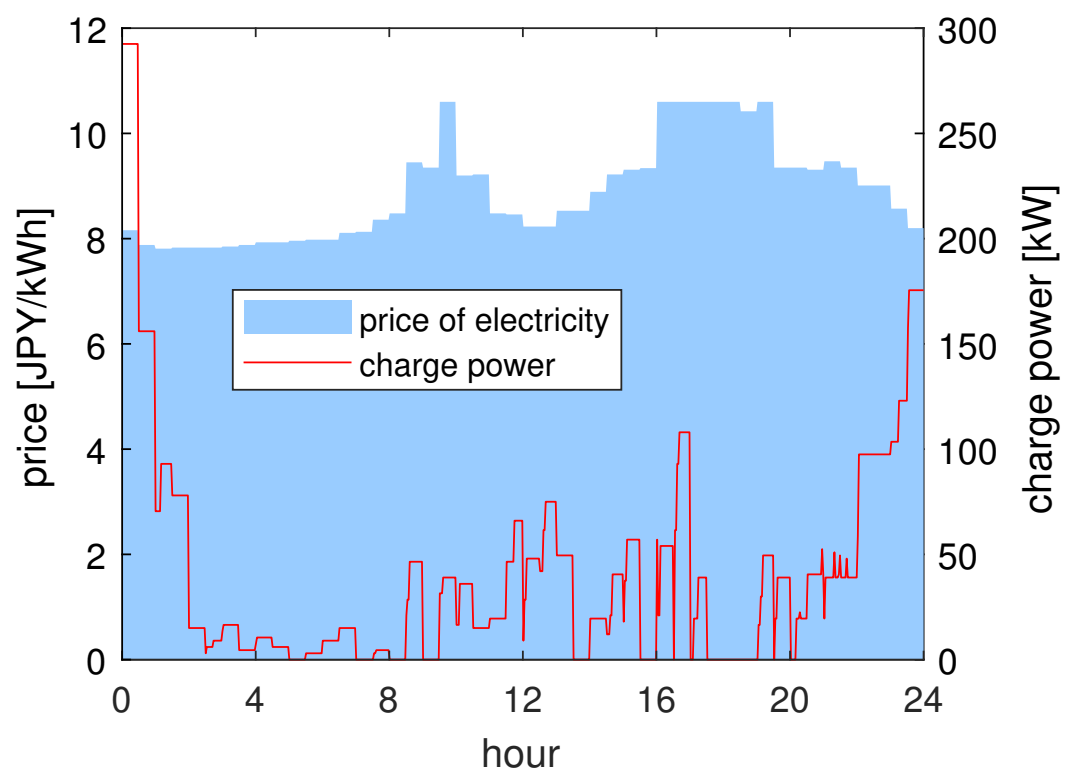

Figure 15: Aggregated charging power and electricity price for case 3 (2-layer scheduling) for one of the real price profiles in the Tokyo wholesale electricity market in $201 \%$.

lower costs at the expense of longer peak waiting times, in the case of very high variability profiles the imperfect information of the model given by the limited optimization horizon can give rise to lower waiting times. This is due to stochastic variability of the results under varying conditions. This effect is particularly evident for results such as peak wait time, which are very sensitive to random variations (one outlier trip can change the result for the whole simulation).

It should be noted that there is not much difference between the unscheduled model and the 1-layer model in terms of costs. This shows that the 1-layer model is unable to effectively optimize charging due to the limited 


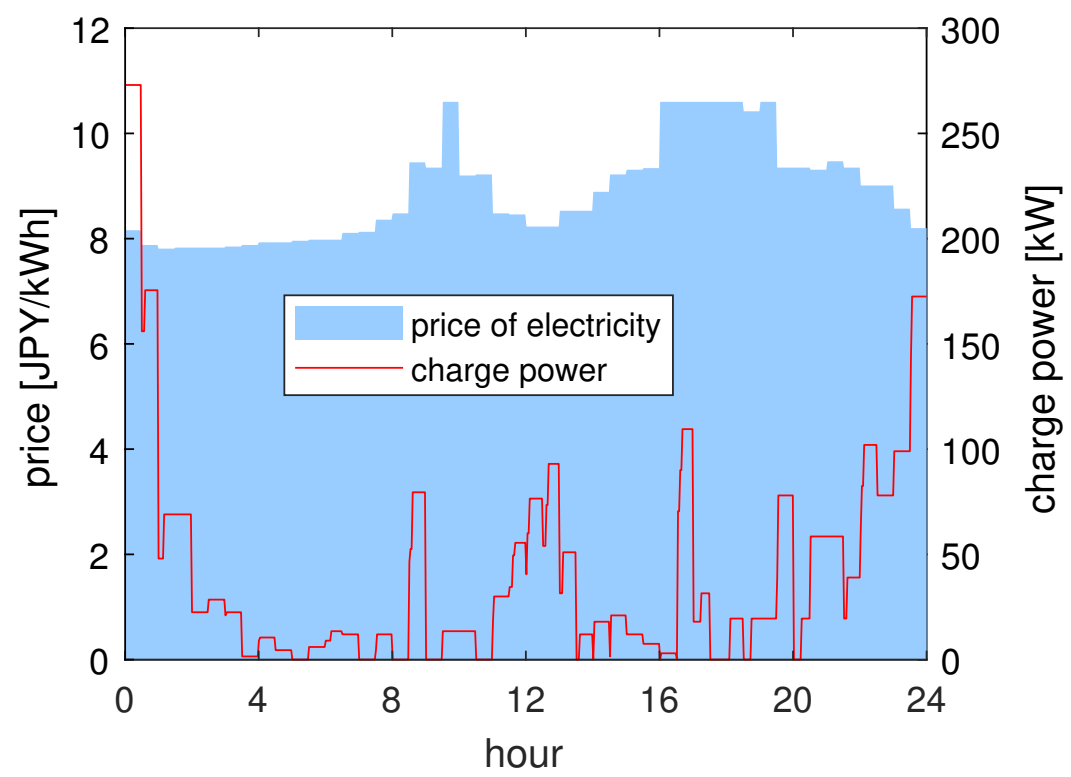

Figure 16: Aggregated charging power and electricity price for case 4 (2-layer scheduling with V2G) for one of the real price profiles in the Tokyo wholesale electricity market in $201 \%$.

optimization horizon. This is evident from Figs. 8 and 9, showing aggregated charging power for the 2 models. The results are almost indistinguishable. The same can be seen for the SOC in Fig. 12. This is in contrast with Figs. 10 and 11, where the charging optimization is evident as the aggregate charging peaks are correlated with lows in the electricity price profile. The state of charge of vehicles during the simulation is shown in Figs. 13 and 14.

Figs. 15 and 16 report aggregate charging levels for a JEPX price profile. Results shown are for the most representative day of the 10 selected, representing a equivalent probability of $68 \%$. It is evident that the price profile is extremely flat, with a maximum price differential of about 2 yen $/ \mathrm{kWh}$. This 


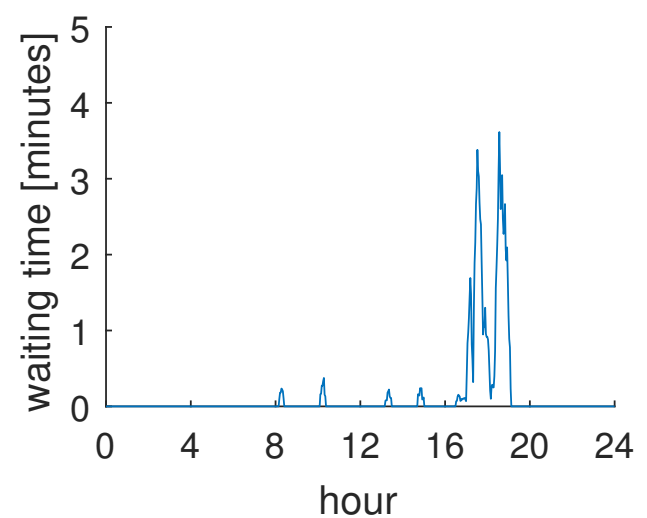

(a)

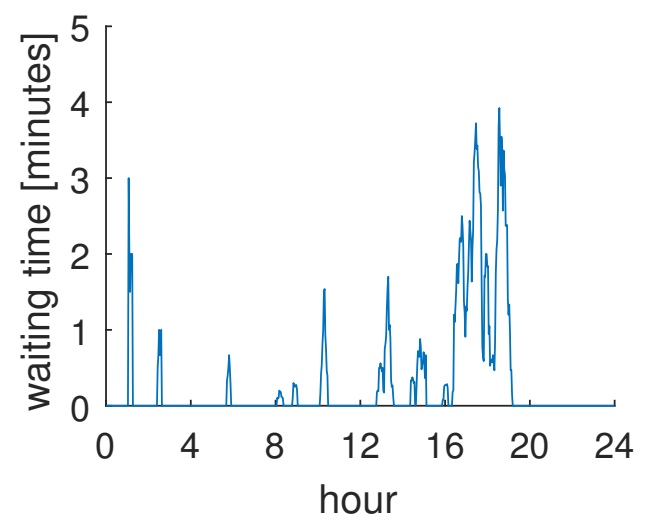

(c)

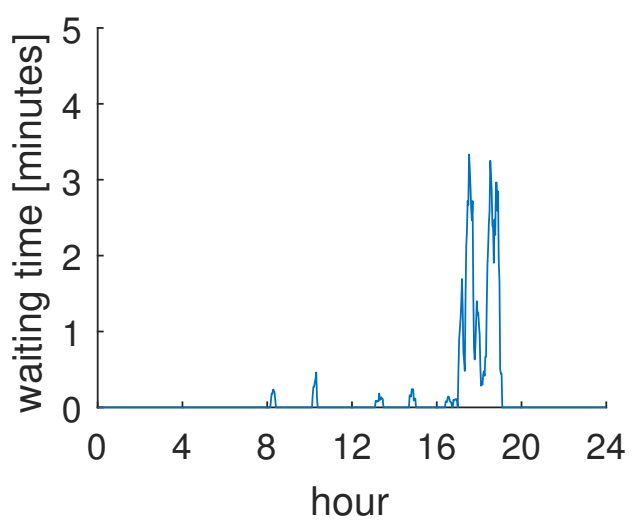

(b)

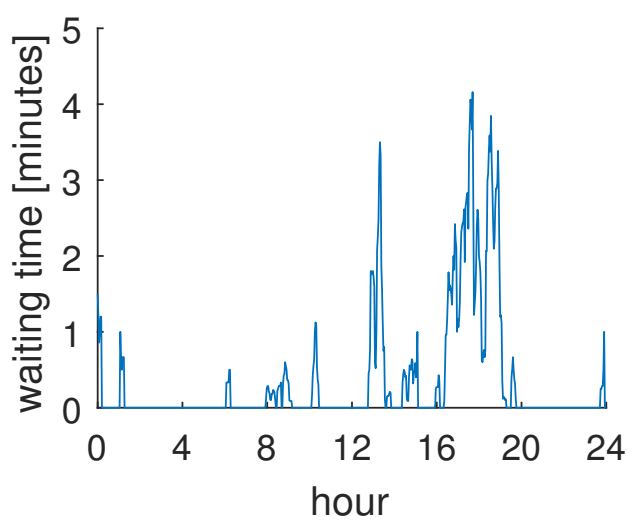

(d)

Figure 17: 10 minutes moving average wait times: (a) case 1; (b) case 2; (c) case 3; (d) case 4 . 


\begin{tabular}{|c|c|c|c|c|}
\hline & 1 & 2 & 3 & 4 \\
\hline \multicolumn{5}{|c|}{ JEPX price profiles } \\
\hline peak wait time (minutes) & 14 & 18 & 21 & 31 \\
\hline 10-min MA peak wait (min.) & $3: 37$ & $3: 23$ & 4:02 & $4: 12$ \\
\hline total electricity cost (yen) & 10493 & 10538 & 9393 & 9282 \\
\hline \multicolumn{5}{|c|}{ gamma-distributed price profiles } \\
\hline peak wait time (minutes) & 14 & 18 & 25 & 21 \\
\hline 10-min MA peak wait (min.) & $3: 37$ & $3: 32$ & $4: 27$ & $4: 23$ \\
\hline total electricity cost (yen) & 25868 & 25218 & 18542 & 14684 \\
\hline
\end{tabular}

Table 4: Wait times and charging costs for the 4 models with the selected scenarios for the Japan Electric Power Exchange in 2017 and with the randomly generated electricity price profiles. MA is moving average.

limits the effectiveness of the charge scheduling algorithm, especially since its priority is transport service. The 10-minutes moving average waiting times for new arrivals for the 4 models is shown in Fig. 17 for the same price profile. The results show new peaks in waiting times corresponding to a low electricity price around noon, since some of the vehicles have been scheduled to charge and avoid the afternoon price peak.

The median computation time for a time step of the 2-layer model on a quad-core $3 \mathrm{GHz}$ Intel Core i5 processor with $32 \mathrm{~GB}$ of RAM was about 20 seconds for the upper layer and 1.5 seconds for the main layer. The average overall was 29.4 seconds per time step ( 5 hours 53 minutes per simulation). 


\section{Conclusions and future work}

An extension of the model in Zhang et al. (2016) was developed to incorporate electricity price information for optimizing vehicle charging. A 2-layer optimization was developed to optimize both charging and transport service at different time scales. The proposed model was applied to a case study in Tokyo, using transport survey data and historical electricity prices from the wholesale electricity market. The results show that the optimization can reduce the costs of charging for the system by $10 \%$ when using historical price profiles from the Japan Electric Power Exchange. Much larger cost savings could be obtained with price profiles with higher variability. With prices sampled from a gamma distribution the savings compared to the unscheduled model are $43 \%$ and $28 \%$ for the case with and without V2G, respectively. The 2-layer model is shown to be superior to the 1-layer model, with the latter being effectively same as the unscheduled model due to the limited time horizon.

Waiting times are not excessively affected by the charging optimization, with 10-minutes moving average wait times peak increasing for the model with V2G by $16 \%$ and $21 \%$ for the real electricity prices and the generated price profiles, respectively, compared to the unscheduled model.

This work shows the potential of SAEV to offer effective energy storage to the grid and avoid grid congestion thanks to dynamic pricing. Several other aspects are open to investigation in future work. An important extension of the model could be the possibility of selection of which nodes are charging stations and which charging stations are available at any given time, for example due to grid constraints or vehicle congestion. In this work only 
wholesale electricity market participation was considered. However, other ancillary services such as operating reserve and frequency regulation could also be provided by the system. These are generally better remunerated and suitable for batteries, thus they are likely more attractive for SAEVs. The potential for the system to provide these services could be investigated by including them in the global optimization.

\section{Acknowledgments}

The authors would like to thank the Ministry of Land, Infrastructure, Transport and Tourism of Japan for the access to the transport survey data.

\section{References}

Acquaviva, F., Paola, D. D., Rizzo, A., Nov. 2014. A novel formulation for the distributed solution of load balancing problems in mobility on-demand systems. In: 2014 International Conference on Connected Vehicles and Expo (ICCVE). pp. 906-911.

Bauer, G. S., Greenblatt, J. B., Gerke, B. F., Apr. 2018. Cost, Energy, and Environmental Impact of Automated Electric Taxi Fleets in Manhattan. Environmental Science \& Technology 52 (8), 4920-4928.

Biondi, E., Boldrini, C., Bruno, R., Sep. 2016. The impact of regulated electric fleets on the power grid: The car sharing case. In: 2016 IEEE 2nd International Forum on Research and Technologies for Society and Industry Leveraging a better tomorrow (RTSI). pp. 1-6. 
Birrell, S. A., Wilson, D., Yang, C. P., Dhadyalla, G., Jennings, P., 2015. How driver behaviour and parking alignment affects inductive charging systems for electric vehicles. Transportation Research Part C: Emerging Technologies 58, 721-731.

Bischoff, J., Maciejewski, M., Jan. 2014. Agent-based Simulation of Electric Taxicab Fleets. Transportation Research Procedia 4, 191-198.

Boyac1, B., Zografos, K. G., Geroliminis, N., Feb. 2015. An optimization framework for the development of efficient one-way car-sharing systems. European Journal of Operational Research 240 (3), 718-733.

Chen, T., Zhang, B., Pourbabak, H., Kavousi-Fard, A., Su, W., Jul. 2018. Optimal Routing and Charging of an Electric Vehicle Fleet for HighEfficiency Dynamic Transit Systems. IEEE Transactions on Smart Grid $9(4), 3563-3572$.

Chen, T. D., Kockelman, K. M., Hanna, J. P., Dec. 2016. Operations of a shared, autonomous, electric vehicle fleet: Implications of vehicle \& charging infrastructure decisions. Transportation Research Part A: Policy and Practice 94, 243-254.

Chen, Z., Liu, W., Yin, Y., 2017. Deployment of stationary and dynamic charging infrastructure for electric vehicles along traffic corridors. Transportation Research Part C: Emerging Technologies 77, 185-206.

Conejo, A. J., Carrión, M., Morales, J. M., 2010. Decision making under uncertainty in electricity markets. Vol. 1. Springer. 
Daina, N., Sivakumar, A., Polak, J. W., Aug. 2017. Electric vehicle charging choices: Modelling and implications for smart charging services. Transportation Research Part C: Emerging Technologies 81, 36-56.

Dallinger, D., Wietschel, M., 2012. Grid integration of intermittent renewable energy sources using price-responsive plug-in electric vehicles. Renewable and Sustainable Energy Reviews 16 (5), 3370-3382.

URL https://econpapers.repec.org/article/eeerensus/v_3a16_ 3ay_3a2012_3ai_3a5_3ap_3a3370-3382.htm

Dong, J., Liu, C., Lin, Z., Jan. 2014. Charging infrastructure planning for promoting battery electric vehicles: An activity-based approach using multiday travel data. Transportation Research Part C: Emerging Technologies 38, 44-55.

Fagnant, D. J., Kockelman, K. M., Mar. 2014. The travel and environmental implications of shared autonomous vehicles, using agent-based model scenarios. Transportation Research Part C: Emerging Technologies 40, 113.

Farhan, J., Chen, T. D., Aug. 2018. Impact of ridesharing on operational efficiency of shared autonomous electric vehicle fleet. Transportation Research Part C: Emerging Technologies 93, 310-321.

URL https://linkinghub.elsevier.com/retrieve/pii/ S0968090X18305758

Freund, D., Raab, A. F., Küster, T., Albayrak, S., Strunz, K., 2012. Agentbased integration of an electric car sharing fleet into a smart distribution 
feeder. In: Innovative Smart Grid Technologies (ISGT Europe), 2012 3rd IEEE PES International Conference and Exhibition on. IEEE, pp. 1-8.

Greenblatt, J. B., Saxena, S., Jul. 2015. Autonomous taxis could greatly reduce greenhouse-gas emissions of US light-duty vehicles. Nature Climate Change 5 (9), 860-863.

He, F., Yin, Y., Zhou, J., Nov. 2015. Deploying public charging stations for electric vehicles on urban road networks. Transportation Research Part C: Emerging Technologies 60, 227-240.

Hörl, S., 2016. Implementation of an autonomous taxi service in a multimodal traffic simulation using MATSim.

URL http://studentarbeten. chalmers.se

Hu, J., Morais, H., Sousa, T., Lind, M., Apr. 2016. Electric vehicle fleet management in smart grids: A review of services, optimization and control aspects. Renewable and Sustainable Energy Reviews 56, 1207-1226.

Iacobucci, R., McLellan, B., Tezuka, T., Jun. 2018. Modeling shared autonomous electric vehicles: Potential for transport and power grid integration. Energy.

JEPX, 2017. Trading Information: Spot Market / Intraday Market.

URL http://www.jepx.org/english/market/index.html

Kang, N., Feinberg, F. M., Papalambros, P. Y., Oct. 2016. Autonomous Electric Vehicle Sharing System Design. Journal of Mechanical Design 139 (1), 011402. 
Levin, M. W., Kockelman, K. M., Boyles, S. D., Li, T., Jul. 2017. A general framework for modeling shared autonomous vehicles with dynamic network-loading and dynamic ride-sharing application. Computers, Environment and Urban Systems 64, 373-383.

Li, S., Huang, Y., Mason, S. J., 2016. A multi-period optimization model for the deployment of public electric vehicle charging stations on network. Transportation Research Part C: Emerging Technologies 65, 128-143.

Liu, J., Kockelman, K. M., Boesch, P. M., Ciari, F., Nov. 2017. Tracking a system of shared autonomous vehicles across the Austin, Texas network using agent-based simulation. Transportation 44 (6), 1261-1278.

Liu, L., Kong, F., Liu, X., Peng, Y., Wang, Q., Nov. 2015. A review on electric vehicles interacting with renewable energy in smart grid. Renewable and Sustainable Energy Reviews 51, 648-661.

Liu, Y., Bansal, P., Daziano, R., Samaranayake, S., Oct. 2018. A framework to integrate mode choice in the design of mobility-on-demand systems. Transportation Research Part C: Emerging Technologies. URL https://linkinghub.elsevier.com/retrieve/pii/ S0968090X18313718

Loeb, B., Kockelman, K. M., Liu, J., 2018. Shared autonomous electric vehicle (SAEV) operations across the Austin, Texas network with charging infrastructure decisions. Transportation Research Part C: Emerging Technologies 89 . 
Marmaras, C., Xydas, E., Cipcigan, L., Jul. 2017. Simulation of electric vehicle driver behaviour in road transport and electric power networks. Transportation Research Part C: Emerging Technologies 80, 239-256.

Ministry of Land, Infrastructure, Transport and Tourism, Japan (MLIT), 2008. Tokyo Metropolitan Area Person trip survey.

Mukherjee, J. C., Gupta, A., Dec. 2015. A Review of Charge Scheduling of Electric Vehicles in Smart Grid. IEEE Systems Journal 9 (4), 1541-1553.

Mwasilu, F., Justo, J. J., Kim, E.-K., Do, T. D., Jung, J.-W., Jun. 2014. Electric vehicles and smart grid interaction: A review on vehicle to grid and renewable energy sources integration. Renewable and Sustainable Energy Reviews 34, 501-516.

Pavone, M., Smith, S. L., Frazzoli, E., Rus, D., Jun. 2012. Robotic load balancing for mobility-on-demand systems. The International Journal of Robotics Research 31 (7), 839-854.

Pourazarm, S., Cassandras, C. G., Wang, T., Apr. 2016. Optimal routing and charging of energy-limited vehicles in traffic networks: Optimal routing and charging of energy-limited vehicles in traffic networks. International Journal of Robust and Nonlinear Control 26 (6), 1325-1350.

Richardson, D. B., Mar. 2013. Electric vehicles and the electric grid: A review of modeling approaches, Impacts, and renewable energy integration. Renewable and Sustainable Energy Reviews 19, 247-254.

Riemann, R., Wang, D. Z. W., Busch, F., Sep. 2015. Optimal location of wireless charging facilities for electric vehicles: Flow-capturing location 
model with stochastic user equilibrium. Transportation Research Part C: Emerging Technologies 58, 1-12.

Spieser, K., Treleaven, K., Zhang, R., Frazzoli, E., Morton, D., Pavone, M., 2014. Toward a Systematic Approach to the Design and Evaluation of Automated Mobility-on-Demand Systems: A Case Study in Singapore. In: Meyer, G., Beiker, S. (Eds.), Road Vehicle Automation. Springer International Publishing, Cham, pp. 229-245.

Tokyo Metropolitan Government, 2016. Tokyo Statistical Yearbook 20092015 .

URL http://www . toukei.metro.tokyo.jp/tnenkan/tn-eindex.htm

Tu, W., Li, Q., Fang, Z., Shaw, S.-l., Zhou, B., Chang, X., Apr. 2016. Optimizing the locations of electric taxi charging stations: A spatial-temporal demand coverage approach. Transportation Research Part C: Emerging Technologies 65, 172-189.

Vahidi, A., Sciarretta, A., Oct. 2018. Energy saving potentials of connected and automated vehicles. Transportation Research Part C: Emerging Technologies 95, 822-843.

URL https://linkinghub.elsevier.com/retrieve/pii/ S0968090X18305199

Volkov, M., Aslam, J., Rus, D., 2012. Markov-based redistribution policy model for future urban mobility networks. In: Intelligent Transportation Systems (ITSC), 2012 15th International IEEE Conference on. IEEE, pp. $1906-1911$. 
Wang, H., Cheu, R., 2013. Operations of a taxi fleet for advance reservations using electric vehicles and charging stations. Transportation Research Record: Journal of the Transportation Research Board (2352), 1-10.

Waraich, R. A., Galus, M. D., Dobler, C., Balmer, M., Andersson, G., Axhausen, K. W., 2013. Plug-in hybrid electric vehicles and smart grids: Investigations based on a microsimulation. Transportation Research Part C: Emerging Technologies 28, 74-86.

Weiss, J., Hledik, R., Lueken, R., Lee, T., Gorman, W., Dec. 2017. The electrification accelerator: Understanding the implications of autonomous vehicles for electric utilities. The Electricity Journal 30 (10), 50-57.

Yabe, T., 2015. Road Network Today.

URL http://www.nikkoken.or.jp/pdf/publication/2015e/2015e3. $\operatorname{pdf}$

Yi, Z., Shirk, M., Jan. 2018. Data-driven optimal charging decision making for connected and automated electric vehicles: A personal usage scenario. Transportation Research Part C: Emerging Technologies 86, 37-58.

Yilmaz, M., Krein, P. T., May 2013. Review of Battery Charger Topologies, Charging Power Levels, and Infrastructure for Plug-In Electric and Hybrid Vehicles. IEEE Transactions on Power Electronics 28 (5), 2151-2169.

Zhang, R., Rossi, F., Pavone, M., May 2016. Model predictive control of autonomous mobility-on-demand systems. In: 2016 IEEE International Conference on Robotics and Automation (ICRA). pp. 1382-1389. 